Article

\title{
Service-Oriented Cooperation Policies for Intelligent Ground Vehicles Approaching Intersections
}

\author{
Kailong Zhang ${ }^{1, *(\mathbb{D})}$, Ce Xie ${ }^{1}$, Yujia Wang ${ }^{1}$ (D), Min Wang ${ }^{1}$, Arnaud de La Fortelle ${ }^{2}$, \\ Weibin Zhang ${ }^{3}$ and Zongtao Duan ${ }^{4}$ \\ 1 School of Computer, Northwestern Polytechnical University, Xi'an 710029, Shaanxi, China; \\ xiece1@mail.nwpu.edu.cn (C.X.); wangyj829@163.com (Y.W.); wangmin316@foxmail.com (M.W.) \\ 2 Centre for Robotics, Mines ParisTech, 75020 Paris, France; arnaud.de_la_fortelle@mines-paristech.fr \\ PATH, UC Berkeley, Berkeley, CA 94804, USA; wbzhang@berkeley.edu \\ 4 School of Information Engineering, Chang'an University, Xi'an 710064, Shaanxi, China; ztduan@chd.edu.cn \\ * Correspondence: kl.zhang@nwpu.edu.cn; Tel.: +86-135-7220-3560
}

Received: 28 August 2018; Accepted: 11 September 2018; Published: 13 September 2018

\begin{abstract}
With the coming of intelligent vehicles and vehicular communication, Intelligent Transportation Systems (ITS) of connected vehicles are emerging and now evolving to Cooperative-ITS (C-ITS), as service platforms for smart cities. Considering new service properties, the autonomous cooperation of such vehicles has exhibited novel QoS features that imply new requirements: guaranteeing the traffic efficiency of any emergent vehicle while trying to promote the throughput at an intersection. So, after analyzing the classic reservation-based cooperation mechanisms, new QoS-oriented cooperation methods and policies are studied in this work. Concretely, several models of related traffic objects we have proposed are firstly introduced briefly. Then, the scheduling policies of vehicles approaching an intersection have been presented, including three existing policies (FAFP-SV, FAFP-SQ, and HQEP-SV) and five new polices (FAFP-SQ-SV, FAFP-MQ, HWFP-SQ, HWFP-SQ-SV, HWFP-MQ). These policies combine two major factors: vehicular priority for scheduling and concurrency in traffics. The first one includes the vehicular arrival-time, priority mapped to QoS, and the weight of reserved vehicles on a lane etc. In addition, the second refers to schedule a platoon rather than single vehicle each time, or platoons on different lanes instead of one platoon on only one lane. All these policies have been implemented, and further, verified within the parameter-configurable traffic simulator QoS-CITS (v2.1) we designed and developed with C\#. Abundant experiments have been conducted with configured typical traffic scenes, and experimental results show that HWFP-SQ-SV and HWFP-MQ can guarantee both the QoS of emergent vehicles and traffic throughput better than other six policies.
\end{abstract}

Keywords: Cooperative-ITS; intelligent vehicles; intersection; quality of service; policy; simulation

\section{Introduction}

Along with the increasing maturation of technologies such as autonomous driving and vehicular communication, nowadays, novel transportation scenarios can be increasingly envisioned that any connected intelligent vehicle traveling on the road could drive itself automatically to its destination, adjusting its behavior adaptively according to the surrounding traffic environment and optimizing its route dynamically under the cloud-based guidance. In addition, it can be also imagined that such vehicles will play more roles as mobile carriers for the future smart cities, not only as private vehicles and taxies mentioned usually, but also as shuttle buses, ambulances, fire-fighting vehicles and others. Under such complicated situations, adaptive and autonomous cooperation has been becoming one distinctive feature of connected intelligent vehicles, and meanwhile, one vital problem for the 
emerging stage of Intelligent Transportation Systems (ITS): Cooperative-ITS (C-ITS), and also, other similar swarm intelligent systems. As predicted in many important research projects, for instance the PATH program of USA, the European Union project AutoNet2030 etc. [1], V2X communication-based cooperation of intelligent vehicles is deservedly the main evolving trend for C-ITS, particularly for large-scale C-ITS because there are more advantages compared with current ITS, more perceptive, more efficient and safer.

Considering these new characteristics, in the domain of C-ITS, a series of models and mechanisms for communication-based inter-vehicle cooperation have been studied, and obvious progress has been achieved in recent decades along with increasing interest from more researchers [2-5]. Based on the capability of Vehicle-to-Infrastructure/Agent (V2I/V2A) and Vehicle-to-Vehicle (V2V) communication, such cooperation problems have been mainly reduced to basic sub-problems [6], typically two as shown in Figure 1. Figure 1a shows the vehicular cooperation at a crossroad. It corresponds to a problem called Passing-Through Intersection (PTI) [7], which mainly focuses on how to guarantee vehicles pass through an intersection safely and efficiently $[8,9]$. And Figure $1 \mathrm{~b}$ indicates the cooperation of vehicles when taking lane-changing or over-taking actions on roads [10]. Nevertheless, when considering service properties of future C-ITS, the connotations of these cooperation problems will become different from classic ones [11-14], and consequently, current mechanisms will be greatly challenged by the following issues.

- How to guarantee the traveling efficiency of emergent vehicles, as well as the traditional traffic safety and throughput?

- How to manage vehicular behaviors synergistically in the complicated spatial-temporal serviced domain?

- What novel cooperative policies will satisfy vehicular service properties?

- How to verify new service-oriented features and designs in large-scale C-ITS environments?

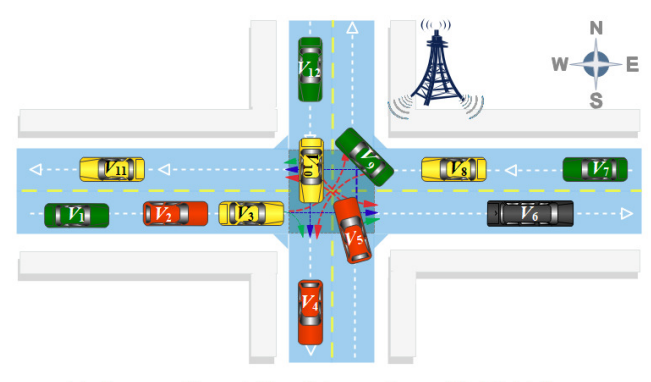

(a) Cooperation at One Intersection with Eight Lanes

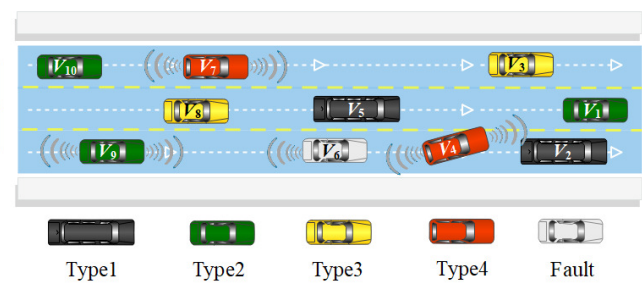

(b) Cooperation on Three Straight-going Lanes

Figure 1. Typical V2X-based Cooperative Situations of Intelligent Vehicles. (a) Cooperation at One Intersection with Eight Lanes. (b) Cooperation on Three Straight-going Lanes.

To explain the differences, here we analyze this problem with a scene in Figure 1a. In this scene, there are four different types of service-oriented vehicles, concretely, type 1: trunk vehicles $V_{6}$; type 2: taxi vehicles $V_{1}, V_{7}, V_{9}$, and $V_{12}$; type 3: bus vehicles $V_{3}, V_{8}, V_{10}$, and $V_{11}$; type 4: ambulance vehicles $V_{2}, V_{4}$, and $V_{5}$. All these vehicles can be reduced to three common service properties: type 4 corresponds to the most emergent degree $\mathrm{H}$ (Highest priority), type 3 has a lower urgency degree $\mathrm{M}$ (Middle priority), type 1 and type 2 own no urgency, corresponding to L (Lowest priority), respectively. In the reality, the higher the priority of one vehicle, the faster this vehicle should pass through an intersection. However, through current methods all these approaching vehicles will cooperate mainly according to the arrival time of each one, equivalent to "First Arrive, First Pass" for both vehicle-vehicle cooperation situations and platoon cooperation situations, which surely leads to emergent vehicles not being able to take any preemptive opportunity. For example, vehicles $V_{2}$ and $V_{5}$ in Figure $1 \mathrm{a}$ are blocked and delayed by middle-emergent vehicles $V_{3}$ and $V_{10}$, separately. It is obvious that such a situation is unreasonable and has violated realities seriously. 
Therefore, how to make vehicles with higher priorities pass through an intersection as early as possible has become a vital problem, which is also the topic with which we have concerned ourselves in recent years. In this paper, several cooperation models, mechanisms, and policies for intelligent vehicles approaching intersections are further studied on the basis of our previous studies $[5,7,15,16]$. Especially, after mapping vehicular QoS to a priority property and studying dynamic priority mechanisms, in this research five new scheduling policies are principally proposed and designed with new "queue-based", namely "platoon-based", and "QoS-oriented" properties. Then, all these designs are implemented within the new version of one self-designed traffic simulator: QoS-CITS (v2.1) [16]. With this simulator, the performance of this study is verified in a mass of typical traffic scenes.

The rest of this paper is organized as follows. In Section 2, the relevant literature is presented. Considering the fundamental procedure of reservation-based passing-through, models of traffic objects and cooperative behaviors proposed in previous work are introduced briefly in Section 3. In Section 4, the main contribution of this study, namely a series of queue-based and QoS-oriented cooperation algorithms and policies, is expressed in detail. All these designs of this work are verified simulatively in Section 5. Finally, research conclusions are drawn and our ongoing work is sketched out in Section 6.

\section{Related Work and Literature}

In the domain of cooperative intelligent vehicles, autonomous driving and V2X-based cooperation are vital and fundamental technologies, which have captured more and more attention in recent decades. In particular, research work has been carried out from different aspects. From the perspective of methodology, interdisciplinary theories and methods, such as intelligent sensing and control, Agent and Multi-Agent-System (MAS), game theory, deep learning, self-organizing network, big data and cloud, model-driven, and simulative verification etc., have been employed more in pursuit of progress.

\subsection{Autonomous Driving Study}

Perception of the traffic environment is the cornerstone of ITS. Recently, the sensing capabilities of ITS have been largely promoted via the employment of more smart sensors, such as LiDAR, GPS, Inertial Navigation System (INS), Radar, Vision and so on, and emerging vehicular communication technologies, typically DSRC(Dedicated Short Range Communication), LTE-V, the coming 5G network, and also high-resolution maps etc.

Surrounding these developing technologies, the theories and mechanisms of vehicular smart perception have been studied recently [17-25]. With the ability of precise perception, the foundation of autonomous driving has been getting better and better. Especially, decision-making, autonomous control, path-planning and behavior policies are several vital aspects that have been studied and experienced [26-31]. For instance, Furda et al. [32] studied a two-stage real-time decision-making method to improve road safety in city traffic systems, and then optimized the real-time decision-making method for autonomous city vehicles to select the most appropriate maneuver. Hubmann et al. [33] presented an online-capable Partially Observable Markov Decision Process (POMDP) framework for autonomous driving in different environment situations. It provides near optimum solutions for the behavior generation on intersections. Kim [34] formulated a Model Predictive Control (MPC) problem to generate a feasible trajectory for one vehicle, and further proposed several constraints and rules to guarantee coordination safety. Weiskircher et al. [35] proposed a predictive trajectory guidance and control framework that enables the safe operation of autonomous and semiautonomous vehicles considering the constraints of operating in dynamic public traffic. Rafaila et al. [36] and Koga et al. [37] also did similar work. Rosolia et al. [38] designed a two-stage nonlinear nonconvex control approach, including an outer-loop nonlinear MPC and a model based on an inner linear feedback controller, for autonomous vehicles driving during highway cruise conditions. Beaucorps et al. [39] proposed a human-like decision-making algorithm for simple intersections without signals in the presence of another vehicle. All these autonomous driving studies make intelligent ground vehicles traveling on roads increasingly possible. 


\subsection{Communication Based Cooperation at Intersections}

V2X communication-based inter-vehicle coordination at intersections is a hot topic in the domain of C-ITS. Typically, several cooperative communication protocols, such as Ballroom Intersection Protocol (BRIP), CAM/DENM(Cooperative Awareness Message/Decentralized Environmental Notification Message)-based cooperation messages etc. [40,41], and further novel cooperation mechanisms have contributed [12,42-44]. In order to solve the problem of BRIP protocol resulting in potential accidents at intersections for GPS errors or control system failures, Aoki et al. [40] designed a new safe and practical intersection protocol named the Configurable Synchronous Intersection Protocol (CSIP). Mladenovic et al. [45] proposed a paradigm shift based upon a self-organizing and cooperative control framework, in which one vehicle will calculate its velocity and trajectory with others coordinately via V2V communication. Then, two controllers are used to separately manage vehicular approaching maneuvers and regulate vehicle-following procedures. After designing a discrete model of an intersection and V2I communication protocols, Wuthishuwong et al. [13] implemented a safe trajectory generation mechanism to avoid possible collision when autonomous vehicles pass through intersections. Liu et al. [46] proposed a decentralized cooperative adaptive cruise control algorithm for vehicles in the vicinity of intersections (CACC-VI), to improve the throughput of intersections by reorganizing vehicle platoons. Similarly, Campos et al. [47] focused on the traffic coordination problem at traffic intersections and presented a decentralized coordination approach, combining optimal control with model-based heuristics that are suitable for a fast-online implementation. Xu et al. [48] proposed a novel distributed conflict-free cooperation method for multiple connected automated vehicles at unsignaled intersections and eventually designed a distributed controller to stabilize the virtual platoon for conflict-free cooperation at intersections. Elleuch et al. [49] proposed a new system called Cooperative Intersection Collision Avoidance Persistent (CICAP) system, and introduced a new intersection collision avoidance system by means of V2V communication. Li et al. [50] proposed a novel traffic control method based on a genetic algorithm aimed at reducing the time in an isolated intersection without traffic lights. Experiments show that this method can greatly shorten the time delay. The problem of autonomous PTI has increased. All these contributions will establish a good foundation for our study of complicated PTI problems that depend on precise positioning and good $\mathrm{V} 2 \mathrm{~V}$ and V2I communication.

\subsection{Simulation and Verification}

Simulative verification is another vital aspect in the domain of C-ITS because it is impractical to construct a large-scale transportation environment of intelligent vehicles [51]. So, simulation has been widely concerned and adopted when exploring autonomous driving and cooperative mechanisms [52-54]. As is well known, there exists a series of famous traffic simulators, such as SUMO, OMNET++, Veins, MovSim etc. [55,56]. In recent studies, several coordination frameworks and mechanisms for cooperative vehicles have been studied. For instance, Debada et al. [57] proposed a coordination framework for Connected Autonomous Vehicles (CAVs) based on the concept of virtual vehicles. Santos et al. [58] presented a simulator framework for cooperative vehicle systems and also a test case for validation. Tang et al. [59] presented a Micro Intelligent Vehicle (MicroIV)-based cooperative driving hardware simulation platform for simulation of C-ITS applications, especially for collaborative maneuvering. Chen et al. [60] established the driving simulation environment by PreScan and then proposed to use the Rough Set theory to abstract the car-following rules to support the decision-making of autonomous vehicles in the complex and dynamic urban environment. Artunedo et al. [61] proposed a tool to simulate cooperative maneuvers among autonomous vehicles in which virtual and real vehicles can interact conjunctively. Kim et al. [62] proposed an integrated road traffic-network-cloud simulator for V2C connected car services (IsV2C) with a user-friendly GUI. Besides these Model-in-Loop mechanisms, some Hardware-in-Loop testbeds are also constructed and employed for the study of ITS $[63,64]$. All these works shows that simulation is feasible, and these methods are worth learning. 
As analyzed above, existing studies have contributed to remarkable progress. However, few studies have been conducted on cooperation mechanisms in the service domain. Mladenovic et al. [45] carried out a similar study, and proposed a priority-related passing-through mechanism, but this work mainly considered the intelligent adjustment of vehicular trajectory and cooperation between two conflicting vehicles, instead of that we concerned. Fok et al. [63] has studied a group of policies for AIM (Autonomous Intersection Management), but they didn't consider vehicular service properties. Therefore, on the basis of our previous work $[5,7,15,16]$, we will study and present several new QoS-oriented cooperative policies in this paper.

\section{Fundamental Traffic Models and Cooperation Procedure}

Since this work is an extension of our previous work, some related models we have proposed in $[5,7,15,16]$ must be described in brief firstly, helping to understand the work in this paper.

\subsection{Fundamental Traffic Environment Objects}

$\ell$ and $\ell_{c}$ are separately defined as a piecewise lane and the current lane on which one vehicle is traveling. An intersection II is a critical traffic area connecting several crossed lanes. In our previous work, $j^{t h}$ intersection $\mathbb{I}_{j}$ is presented as a tuple: $<i d=j, \zeta_{\ell}^{\mathbb{I}_{j}},\left\{<\ell_{j k}, \omega_{j k}, c_{k 1}, c_{k 2}>\mid \ell_{j k} \in \zeta_{\ell}^{\mathbb{I}_{j}}, k \in\right.$ $\left.\left\{1,2, \ldots,\left|\zeta_{\ell}^{\mathbb{I}_{j}}\right|\right\}\right\}>$, as detailed in Table 1 .

Table 1. Definitions and Parameters of Traffic Object.

\begin{tabular}{|c|c|c|c|}
\hline Para & Definition & Para & Definition \\
\hline \multicolumn{4}{|c|}{ Intersection, Critical Section, and Special Positions for one Intersection } \\
\hline I.id & identification number. & I. $\zeta_{\ell}^{\mathrm{II}}$ & set of connected lanes. \\
\hline $\mathbb{I} . \omega_{j k}$ & direction of $\ell_{j k} ;$ & I. $c_{k 1}$, II. $c_{k 2}$ & coordinates of two connecting \\
\hline$\gamma . i d$ & $\begin{array}{l}\text { I: entering, } \mathrm{U} \text { : leaving. } \\
\text { identification number. }\end{array}$ & $\gamma \cdot \vec{c}$ & $\begin{array}{l}\text { points between } \mathbb{1}_{j} \text { and } \ell_{j k} \text {. } \\
\text { coordinate vector of all vertices. }\end{array}$ \\
\hline$\gamma \cdot \zeta_{\rho}^{k}$ & set of connected lanes. & $\gamma \cdot s$ & status of section: available or forbidden. \\
\hline$\gamma . l$ & length of $\gamma$. & $\gamma . w$ & width of $\gamma$. \\
\hline$\gamma \cdot \tau$ & token of $\gamma$. & $P_{v}$ & vehicular current position. \\
\hline$P_{a}$ & $\begin{array}{l}\text { position where vehicles beginning } \\
\text { to adjust the velocity. }\end{array}$ & $P_{r}$ & $\begin{array}{l}\text { position where vehicles submit } \\
\text { reservation messages. }\end{array}$ \\
\hline$P_{\gamma_{m} b}$ & $\begin{array}{l}\text { position where vehicles start to } \\
\text { decelerate if it can't obtain } \gamma_{m} . \tau \text {. }\end{array}$ & $P_{\gamma_{m} p}$ & $\begin{array}{l}\text { position vehicles can't travel across } \\
\text { when } \gamma_{m} . \tau \text { is not authorized. }\end{array}$ \\
\hline \multicolumn{4}{|c|}{ Model of Heterogenous Vehicles } \\
\hline v.id & identification number. & $v . \xi$ & purpose of vehicle or vehicular mission. \\
\hline v.p & $\begin{array}{l}\text { vehicular priority, corresponding } \\
\text { to its QoS. }\end{array}$ & $\mathbb{E}$ & $\begin{array}{l}\text { classic vehicle-specific physical } \\
\text { and kinematic parameters. }\end{array}$ \\
\hline$v \cdot l$ & current lane. & $v \cdot \gamma$ & current occupied section or null. \\
\hline v.s & current state. & $v \cdot \alpha$ & current action. \\
\hline$v \cdot \vec{\gamma}$ & vector of required S-CSs. & $v \cdot \vec{\tau}$ & vector of required tokens. \\
\hline \multicolumn{4}{|c|}{ Vehicular Action Model } \\
\hline$\alpha . i d$ & identification number. & $\alpha . \zeta_{s}$ & $\begin{array}{l}\left\{s_{0}, \ldots, s_{k-1}\right\}, s_{i} \text { is }(i+1)^{t h} \text { status of } \\
\text { this action. }\end{array}$ \\
\hline$\alpha \cdot M_{S}$ & $\begin{array}{l}\text { action transition matrix: } \\
M_{S}=\left[\varepsilon_{i j}\right]_{k \times k}\end{array}$ & $\alpha \cdot M_{\mathcal{C}}$ & $\begin{array}{l}\text { condition matrix for action transiting: } \\
M_{c}=\left[c_{i j}\right]_{k \times k}\end{array}$ \\
\hline
\end{tabular}

To improve the traffic efficiency, one intersection is always divided into a group of no-overlapping rectangular parts, and each inseparable part can be only allocated to one vehicle each time. In our work, each such part is presented as a Static Critical Section(S-CS): $\gamma$, as shown in Figure 2a. Furthermore, the $m^{\text {th }}$ critical section $\gamma_{m}$ is expressed via a tuple : $\left\langle i d=m, \vec{c}, l, w, \zeta_{\ell}^{m}, \tau\right\rangle$, as detailed in Table 1 . Of course, one intersection can be mapped to a unique critical section as shown in Figure $2 b$, simplifying 
the traffic management while also decreasing the efficiency. Thus, one intersection II can be presented as a relationship set $\zeta_{\gamma}^{\mathbb{I}}$.

In Figure $2 b$, there are also a set of particular positions, $P_{a}, P_{r}$, and $P_{\gamma b}$, which are the foundation of the reservation-based cooperation. Correspondingly, there are also several distance notations, $d_{a}, d_{r}$, and $d_{b}$, employed in our models. All these aforementioned notations are explained in Table 1.

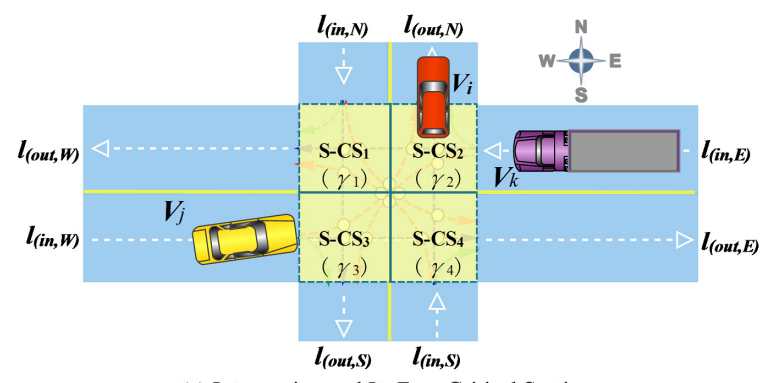

(a) Intersection and Its Four Critical Sections

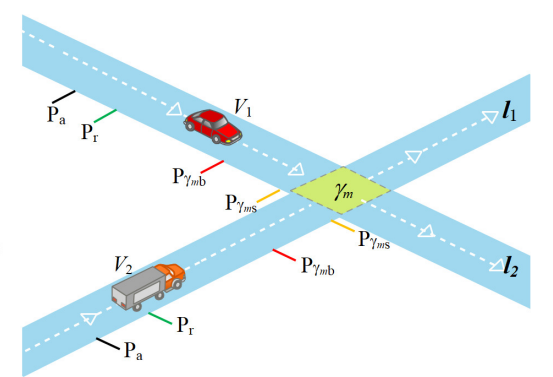

(b) Positions for One Static Critical Section

Figure 2. Intersection and Its Composed S-CSs. (a) Intersection and Its Four Critical Sections.

(b) Positions for One Static Critical Section.

\subsection{Modeling Heterogeneous Intelligent Vehicles and Their Behaviors}

(1) Model of Intelligent Vehicles

Considering that intelligent vehicles serve as diverse mobile carriers in the coming C-ITS, we have designed a vehicular model with service-related properties as a tuple $v_{i}::=\langle i d=i,\langle\xi, p\rangle, \mathbb{E},<$ $\ell, \gamma, s, \alpha>,\langle\vec{\gamma}, \vec{\tau}\rangle>$, as detailed in Table 1 . In this model, service properties are indicated by the first sub-tuple. The classic physical properties are presented by $\mathbb{E}$, including vehicular length (v.l), width $(v . w)$, current acceleration $(v . a)$ and velocity $(v . v)$, length between both axes $v . \Delta l_{w}$, maximum velocity $(v \cdot \bar{v})$, and the kinematic model etc. Current vehicular position and behavior status are indicated by the second sub-tuple, and the reserved vector of S-CS and the corresponding tokens are in the last element. Our previous study has implemented this parameter-customizable model and demonstrated that this model is effective enough.

\section{(2) Reservation-based Vehicular Actions and States}

For the reservation-based passing-through problem, whether one vehicle is allowed to pass through a critical section or not primarily depends on if it can successfully obtain the token of this section. So, vehicular behaviors will be closely related to reservation and authorization. In our previous work, we have abstracted different vehicular motions as a uniform event-triggered action model. Concretely, $i^{\text {th }}$ action $\alpha_{i}$ is presented as a tuple $<i d=i$, name, $\zeta_{s}, M_{s i}, M_{c i}>$. On this basis, a group of PTI-related vehicular actions has been designed, covering Initialization $\alpha_{0}$, Cruise with reservation $\alpha_{1}$, Entering $\alpha_{2}$, Cruise $\alpha_{3}$, Lane-changing $\alpha_{4}$, Overtaking $\alpha_{5}$, Passing $\alpha_{6}$, Parking $\alpha_{7}$, and Termination $\alpha_{8}$. All these proposed actions and their conditions are detailed in [15].

With these new action models, any vehicular behavior can be essentially presented as a scheduling sequence of actions. Consequently, we employed the concept of behavior state $S$ and an event-triggered sate-automata $\mathbb{A}_{S}$ to represent any special maneuver case and any vehicular movement procedure, separately. The automata $\mathbb{A}_{S}$ is composed of an action set $\zeta_{\alpha}^{S}$, an event-based action-transition matrix $M_{A}$ and a state-transition matrix $M_{S}$. After normalizing all possible maneuver stages, eight vehicular states have been designed to present anyone traveling procedure, and the state-switch logic of $\mathbb{A}_{S}$ as shown in Figure 3. More details are presented in $[5,7,15]$. 


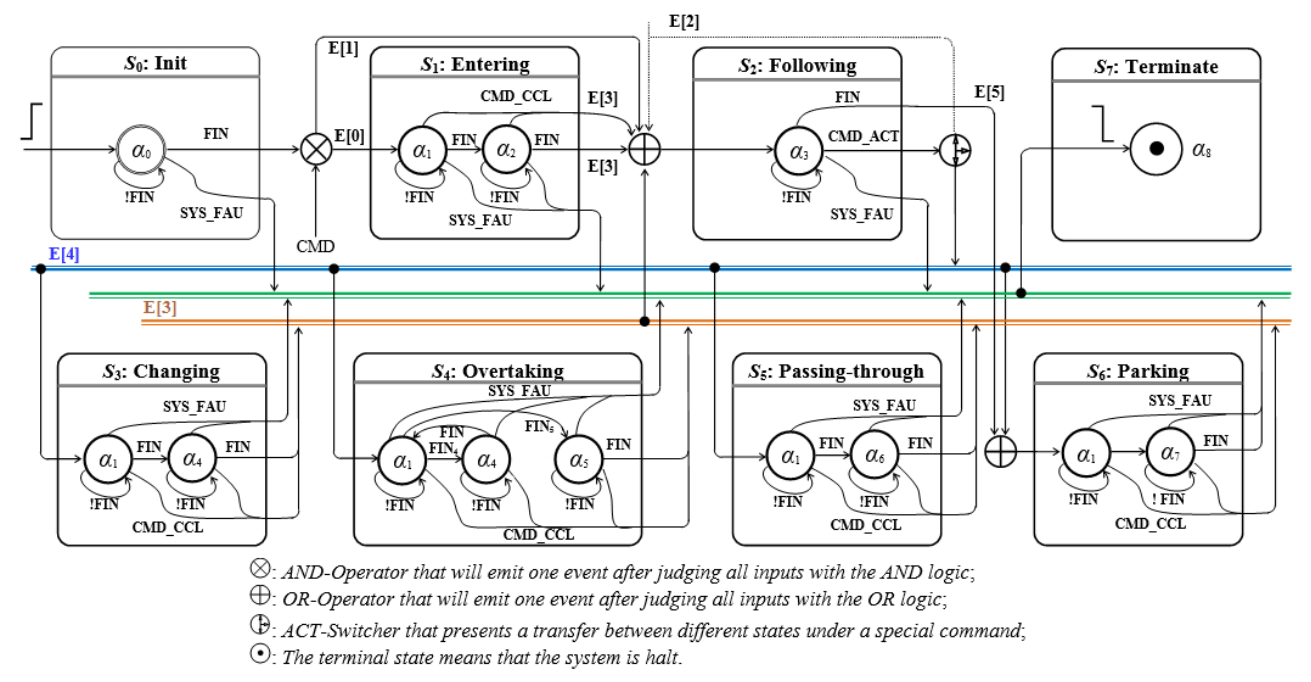

Figure 3. Event-triggered S-Automata $\mathbb{A}_{S}$.

\subsection{Centralized Scheduler: I-Agent}

Under the centralized mode, for an intersection $\mathbb{I}_{j}$, a manager $\mathbb{I}_{j}$-Agent is employed to coordinate traffic flows passing-through each $\gamma_{m}\left(\gamma_{m} \in \zeta_{\gamma}^{\mathbb{I}_{j}}\right)$, scheduling vehicles approaching $\mathbb{I}_{j}$ via particular policies. Structurally, $\mathbb{I}_{j}$-Agent is mainly composed of one traffic-flow scheduler with several policies, and two-level queues to buffer vehicular reserving requirements. Furthermore, a token $\gamma_{m} . \tau$ is employed for authorization and to guarantee traffic flows through $\gamma_{m}$ safely.

It needs to be emphasized that, $Q_{\ell_{k}}$ and $Q_{\gamma_{m}}$ are used to queue reservations from vehicles on lane $\ell_{k}\left(\ell_{k} \in \zeta_{\ell}^{\mathrm{I}_{j}}\right)$ and from those vehicles reserving the critical section $\gamma_{m}$, respectively. Every element of $Q_{\ell_{k}}$ is a vector $<<v \cdot i d, v \cdot x_{c}, v \cdot y_{c}, v \cdot \ell_{c}, v \cdot p>,<v \cdot \gamma_{1}^{\prime}, t_{(s, 1)}, t_{(e, 1)}>,<v \cdot \gamma_{2}^{\prime}, t_{(s, 2)}, t_{(e, 2)}>, \ldots,<$ $v \cdot \gamma_{r}^{\prime}, t_{(s, r)}, t_{(e, r)}>, \cdots>\cdot \gamma_{r}^{\prime}$ presents $r^{\text {th }}$ S-CS in the vector $\vec{\gamma}$ that $v$ applied, and it corresponds to a particular S-CS in $\mathbb{I}_{j}$, e.g., $\gamma_{m}$. $t_{(s, r)}$ and $t_{(e, r)}$ are separately the time when $v$ enters in and leaves from $\gamma_{r}^{\prime}$. Each element in $Q_{\gamma_{m}}$ indicates the queuing information of vehicles that have applied the token of $\gamma_{m}$, and is formalized as a vector $\left\langle v . i d, v \cdot \ell_{c}, t_{s}, t_{w}\right\rangle$, where $t_{w}$ is the time length $v$ has stayed in this queue.

Each vehicle approaching $\mathbb{I}_{j}$ should submit $\mathbb{I}_{j}$-Agent a vector of required S-CSs and related entering time and leaving time of every required S-CS. This time properties have been reduced to be the distance in time domain: Passing-Time-Window $(P T W)$, which is a classic transformation in similar studies. PTW can be further employed to predict potential collisions among vehicles approaching one intersection, and to estimate the approximately delay time for any vehicle. In our research, the Passing-Time-Window of $v_{j}$ through $v_{j} \cdot \gamma_{i}^{\prime}$ is indicated as $P T W_{j i}:=\left[t_{j i, s}(X), t_{j i, e}(Y)\right]$, where $X$ is the first entering point of $v_{j}$ and $Y$ is the last departing point from $\gamma_{i}^{\prime}$. Based on PTW, a group of constraints for safe authorization are detailed in our study [5,7].

\subsection{The Unified Reservation-Based Passing-Through Procedure: RAAL}

With the aforementioned models, all reservation-oriented vehicular behaviors can be represented uniformly as shown in Figure 4 . It is important to note that $\alpha_{i}$ in Figure 4 can be either a single action $\alpha_{6}$ or a sequence of $\alpha_{1}$ and $\alpha_{6}$ relying on the amount of S-CSs $v_{j}$ has been authorized. $\gamma_{k}^{\prime}$ presents the $k^{\text {th }}$ S-CS in the vector $v \cdot \vec{\gamma}$, corresponding to a critical section $\gamma_{m}\left(\gamma_{m} \in \zeta_{\gamma}^{\mathbb{I}}\right)$. Furthermore, we designed a uniform state-based passing-through procedure "Reserve Advance, Act Later" (RAAL) as below.

Step 1. At $P_{a}, v_{i}$ adjusts its velocity to $v_{r}$ in state $S_{2}$.

Step 2. At $P_{r}$, the state of $v_{i}$ switches to $S_{5}$, and $v_{i}$ begins to reserve tokens $v_{i} \cdot \vec{\tau}$. 
Step 3. In $S_{5}$, if $v_{i}$ has gotten the token of its first reserved S-CS $\gamma_{1}^{\prime}$ before $P_{\gamma_{1}^{\prime}}$, it will enter $\gamma_{1}^{\prime}$ at a velocity no more than $v_{\gamma}$ or $v_{r}$, which depends on whether the subsequent tokens are allocated or not. Else, $v_{i}$ must decelerate to wait for $\gamma_{1}^{\prime} \cdot \tau$, and if the token is not authorized $v_{i}$ must stop before $P_{\gamma_{1}^{\prime} p}$.

Step 4 . In $S_{5}$, at the moment when $v_{i}$ leaves from $\gamma_{k}^{\prime}$, it releases $\gamma_{k}^{\prime}$. $\tau$ immediately, and when departing from II the state of $v_{i}$ switches to $S_{2}$.

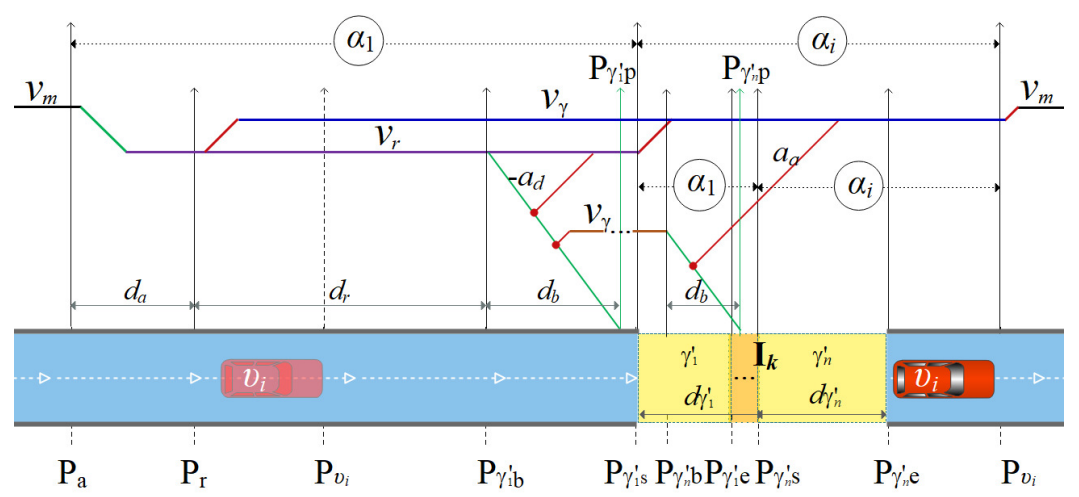

Figure 4. Vehicular Kinematic Features during Passing through S-CSs in the Temporal-Spatial Domain.

\section{Design of Cooperation Policies and Algorithms}

Essentially, authorization policies are the core of cooperative scheduling mechanism, which, as we all known, primarily affects the traffic efficiency. In obvious research, the arrival time has been considered as the basis of scheduling, in other words, vehicular arrival time is the simplest priority. In addition to this, the concept of QoS is rarely concerned. So, after analyzing one basic time-based policy and one QoS-oriented policy we designed, in this section, we further propose several new policies with several new features, to improve the parallelism level of passing vehicles and the concurrency utilization of S-CSs resources. To be clear, vehicles with Highest, Middle, Lowest priorities are marked as vehicles $(\mathrm{H})$, vehicles $(\mathrm{M})$, and vehicles $(\mathrm{L})$ separately in the following parts.

\subsection{Typical Priority-Related Single-Vehicle Policies}

\section{(1) Arrival Time-Based Policy: FAFP}

The main thought of "First-Arrive-First-Pass" policy (FAFP) is taking the arrival time of each vehicle as the unique criterion, namely the priority. It means that the vehicle arriving earlier will hold a higher priority. Concretely, one vehicle with the minimum $t_{(s, 1)}$ will be authorized to pass prior to other vehicles in all $Q_{\ell_{k}}\left(k=0,1, \ldots,\left|\zeta_{\ell}^{\mathbb{I}}\right|\right)$. The procedure of this fundamental time-based cooperation is presented as Algorithm 1. On this basis, FAFP can be further extended with the thought of platoon cooperation, which allow a group of headmost vehicles on one lane be authorized every time, rather than single one.

Although FAFP is the most common policy adopted in current ITS studies for both centralized and distributed cooperation, it has presented obvious weakness in many complicated situations, because of its too simple criterion and design. 


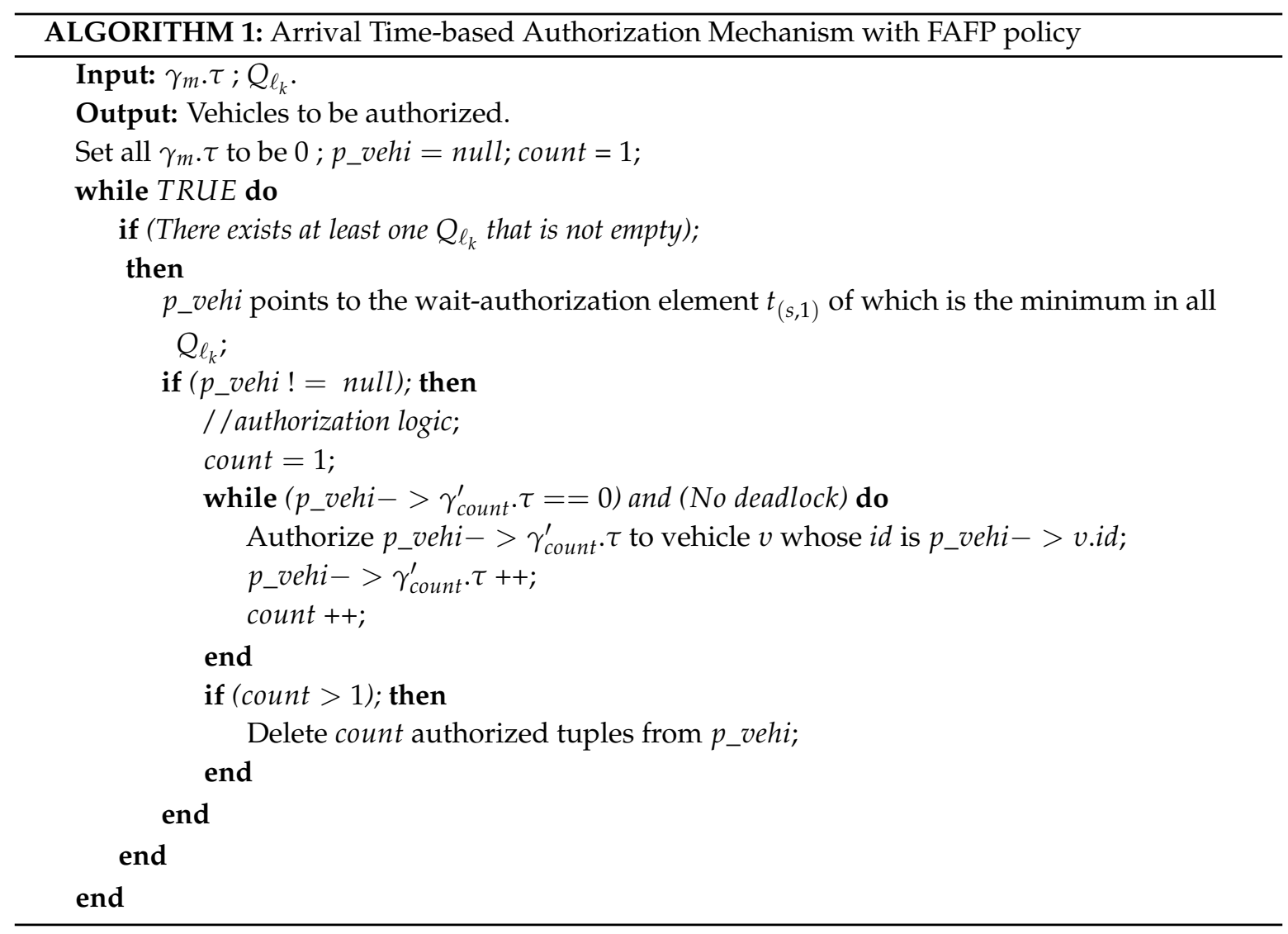

(2) Priority-Inheritance Policy: HQEP

Different from FAFP, “Higher-QoS-Earlier-Pass" policy (HQEP) is the policy that regards vehicular service property as the scheduling criteria. After mapping a QoS level to vehicular priority , the goal of HQEP can be described as: Try the best to guarantee vehicles with highest priorities (vehicles $(\mathrm{H})$ or vehicles (M)) in current situation being authorized preferentially as possible. However, this goal is always challenged under real traffic situations because of the Priority-Inversion-Phenomenon (PIP), where vehicles with higher priorities are always blocked by those vehicles with lower priorities but ahead. Such phenomena are harmful for real transportation systems and daily life, but it is indeed very common.

To resolve this problem and optimize the traffic flow, we have proposed a Gradual-PriorityInheritance (GPI) algorithm in [7], as an instance of HQEP. Its main thought is: If find the first vehicle (H) $v$ has been delayed by vehicles on other lanes, choose the direct precursor vehicle of $v$ on its lane $v \cdot \ell_{c}$ and improve its priority to the highest priority; Iteratively deal with the vehicles ahead of $v$ on $v \cdot \ell_{c}$ one by one, until $v$ is no longer delayed or the precursor vehicle is the head on $v \cdot \ell_{c}$. The HQEP-based cooperation principle can be explained with the Algorithm 2. The advantage of this approach is that when there are few vehicles with the highest priority, the agent will schedule these vehicles with less delay as possible. Meanwhile, such gradual priority inheritance mechanism will allow those ahead vehicles that do not block the vehicles with the highest priority and on other lanes to travel through the intersection normally. Thus, the traffic efficiency at one intersection will be guaranteed as possible. 


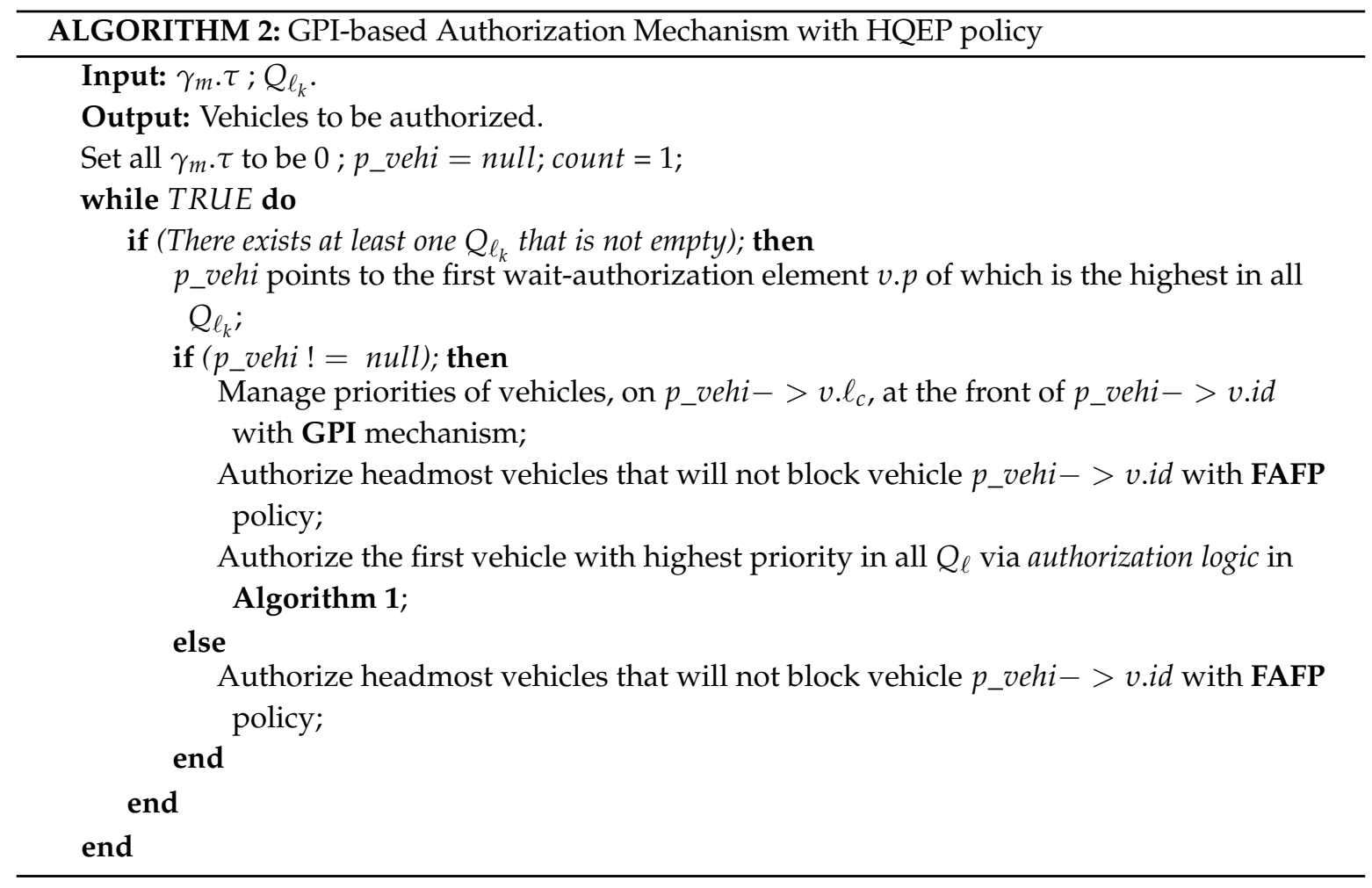

(3) Lane-Weight-Based Policy: HWFP

Considering the "flow" property on any lane, "Highest-Weight-First-Pass" (HWFP) is designed to schedule vehicles according to the status of each lane. The concept "Lane-Weight" is employed to present the priority property of each lane queue, rather than one vehicle. Considering three main properties, namely, the sum of vehicular emergency degrees (or, QoS level), the accumulated delay time, and the arrival time of the first Vehicle $(\mathrm{H})$ on each lane, the Lane-Weight $W_{\ell_{k}}$ of the lane queue $Q_{\ell_{k}}$ can be calculated by the Formula (1). In this formula, we employ the first item to indicate the weight amount of vehicular priorities, where $C(x)$ is the function to count the number of vehicles with the priority $x$ in $Q_{\ell_{k}} ; \varphi_{x}$ is the priority constant of priority $x$, a higher priority corresponds to a bigger constant value. The second item presents the blocked degree of all vehicles in $Q_{\ell_{k}}$. One lane blocked more will get more opportunity to be selected when the first items of different lanes are approximate. The third item is the auxiliary for situations where there are multiple vehicles $(\mathrm{H})$ in different lane queues, and it will help to determine which lane should be selected when the weights of these lanes are equal. Via enlarging the difference of priority constants, different authorization effects can be achieved, for example authorizing vehicles $(\mathrm{H})$ all the time, or giving a breathing space to those lanes without any vehicle $(\mathrm{H})$ but have been heavily blocked, etc. Comparatively, such design will be more flexible to support the QoS-oriented authorization and cooperation.

$$
\left\{\begin{array}{l}
W_{\ell_{k}}=\sum_{x=P_{L}}^{P_{H}}\left(C(x) \times \varphi_{x}\right)+\sum_{x=1}^{\left|Q_{\ell_{k}}\right|} D_{x}+\frac{1}{T_{0}\left(Q_{\ell_{k}}\right)} \\
D_{x}=t_{\text {current }}-t_{(s, 1) \text { of } v_{x}} \\
T_{0}\left(Q_{\ell_{k}}\right)=\left\{\begin{array}{l}
t(s, 1) \text { of } v^{\prime}: \text { if find the first vehicle }(H) v^{\prime} \text { in } Q_{\ell_{k}} . \\
\infty: \text { if not find vehicle }(H) \text { in } Q_{\ell_{k}} .
\end{array}\right.
\end{array}\right.
$$

On this basis, a HWFP-based authorization algorithm for an I-Agent can be briefly explained as: (1) Receive reservation messages from approaching vehicles, and update all queues; (2) Evaluate the weights of all $Q_{\ell}$; (3) Select the queue with the maximum weight, and authorize available required tokens to the head vehicle in this queue. 


\subsection{Enhanced Policies with Parallel Characteristics}

Single vehicle scheduling policies are so simple to realize, but the disadvantages of such policies are also very obvious. The "one-by-one" authorization mechanism will cause the stuttering of vehicular acceleration and traffic flows, and further, heavily affect the traffic efficiency at an intersection. So, besides these aforementioned policies, we further devote to explore some new methods to improve traffic efficiencies through enhancing the parallelism of these policies as far as possible.

(1) Platoon-based Authorization Policy: PAP

The first way is to minimize the stuttering of vehicular acceleration by authorizing a group of vehicles each time, rather than a single one that is the main reason causing the stuttering of vehicular behavior. It can be easily imagined that when one vehicular platoon on a lane is authorized every time, the velocity profiles of these vehicles will probably get smoother than that when authorizing vehicles individually. Many studies has shown that this is an important manner for improving traffic efficiencies.

Based on the traditional platoon method, a Platoon-Authorization Policy (PAP) is designed in this paper to improve the performance of those aforementioned policies. Concretely, for a length setting $N$, the value of every $\gamma . \tau$ with this policy can be maximally $N$, which is equal to the amount of vehicles authorized with this token. In addition, only when the value of $\gamma \cdot \tau$ is zero, $\gamma \cdot \tau$ is usable and it can be authorized to another vehicular platoon. The main mechanism with this policy is described in Algorithm 3.

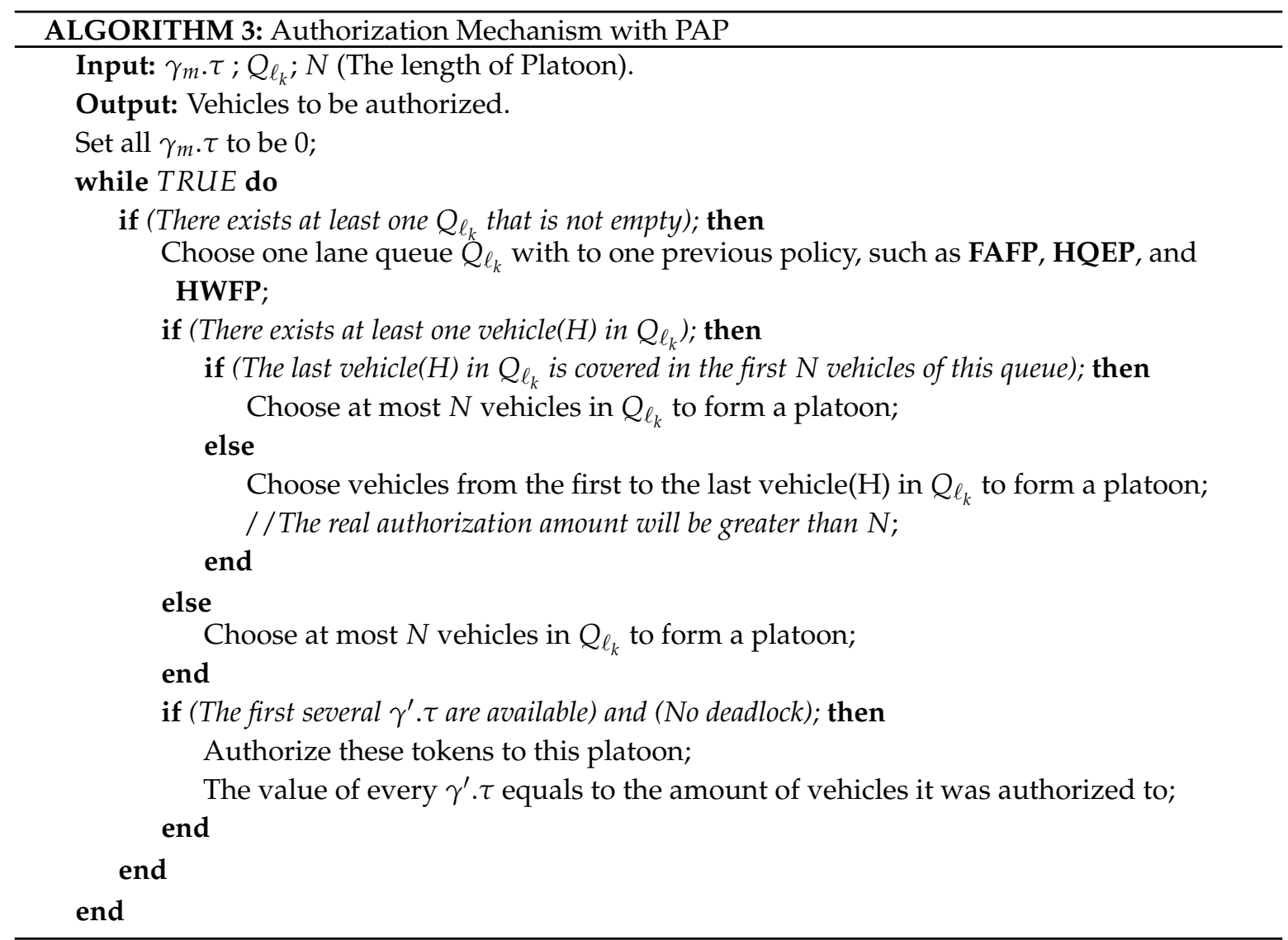

It needs to be emphasized that when merging PAP with above policies, new compound policies will be formed. These new policies will also exhibit different characteristics. Please note that, with the "PAP+HEQP" policy, the real length of one authorized platoon in the Algorithm 3 is possible greater than $N$. This is because we introduced a new consideration that, if the first vehicle $(\mathrm{H})$ will be 
still blocked after the platoon ahead it on the same lane has been scheduled, the platoon should be lengthened to cover this vehicle $(\mathrm{H})$. Which is meaningful to warrant the QoS of this vehicle $(\mathrm{H})$. So, we can regard it as a variant of PAP in a sense.

(2) Multi-Lane Authorization Policy: MLAP

Authorizing one intersection as a whole to one vehicle or one vehicular platoon every time is obviously a very convenient way to manage approaching vehicles. However, we can easily prove that there still exists some opportunity to optimize these policies, in particular, when only a part of $\zeta_{\gamma}^{\mathbb{I}}$ is occupied by one vehicle or platoon the left S-CSs are maybe usable for vehicles on other lanes. In reality, such scenes are very normal. Typically, for example, all vehicles on different lanes are reserving to turn right in $\mathbb{I}$, or vehicles on two parallel lanes are reserving to pass straightly. Under such situations, it should be more reasonable to allow these vehicles on different lanes passing through concurrently.

Based on this knowledge, we propose a new auxiliary policy "Multi-Lane Authorization Policy" (MLAP). Its main idea is described as: Choose one head vehicle or a head platoon from all lanes and authorize this/these vehicles; Check if there are still some free S-CSs after an authorization, if yes, authorize head vehicle(s) on another lane if these free S-CSs construct the mutual head part of $\vec{\gamma}$ of the vehicle(s). The core mechanism is shown in Algorithm 4. Different from the thought of PAP that is used to improve traffic flows by smoothing vehicular velocity profiles, MLAP aims to improve the utilization of multiple physical zones. But essentially, these are all parallel authorization policies, and theoretically should be very beneficial for promoting the throughput of intersections.

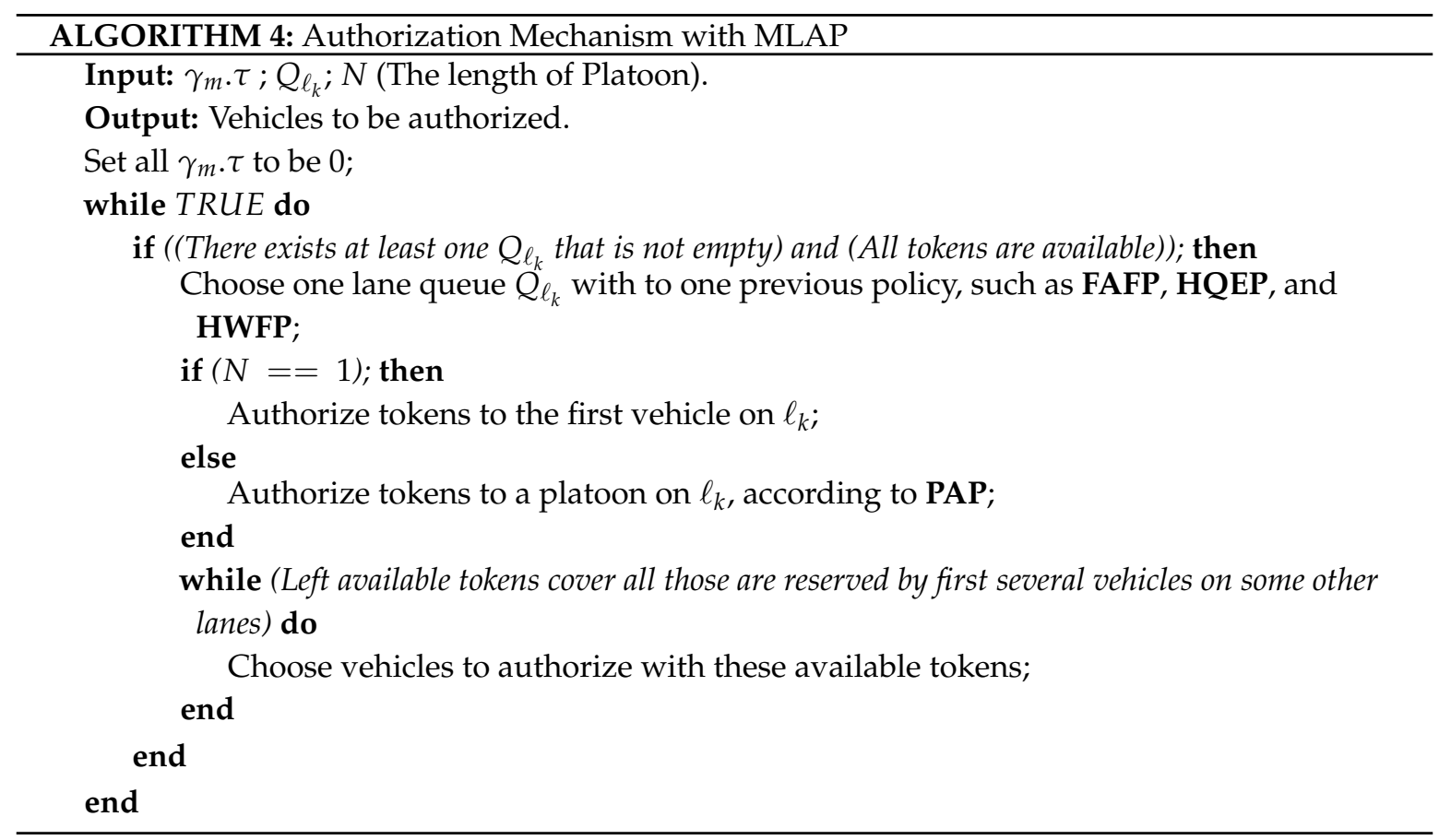

\subsection{Composite Policies: Taking Advantages of Different Ones Above}

To utilize the advantage of different policies, we further composite these different single policies in pairs to improve both the passing-through efficiency of vehicles $(\mathrm{H})$ and the traffic throughput at an intersection. Typically, in this study all eight aforementioned policies have been implemented. In the following, expected effects of these different policies under the same traffic situation are shown in Figure 5 are explained, and as shown in Figures 6 and 7, Figures $6 c, d$ and $7 b-d$ show five new ones. It is important to note that symbols "SQ" and "MQ" in the names of these policies are relevant to the PAP and MLAP, respectively. Although different factors have been considered, the performance of each policy still needs to be further verified. 


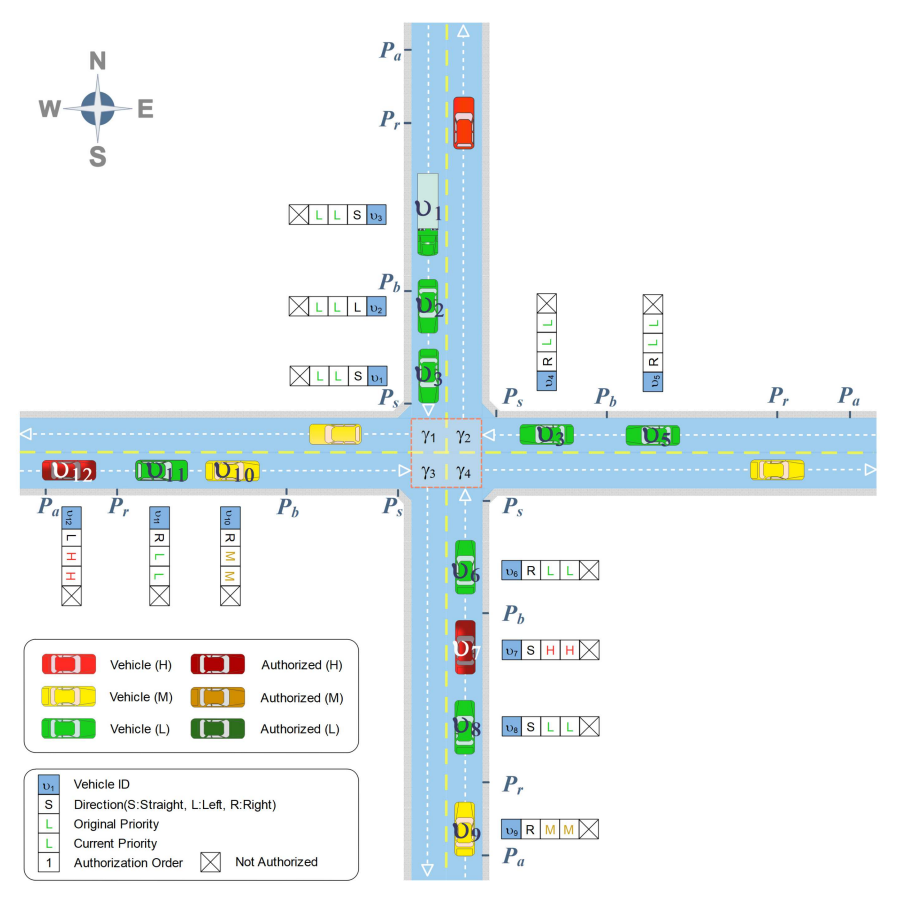

Figure 5. A Traffic Scene.

(1) Four FAFP-Based Policies

a. FAFP-SV: Regard the whole intersection as a single S-CS; When the intersection is free, only choose and authorize the whole intersection to one single vehicle that applies to pass through at the earliest. As shown in Figure 6a, for the first arrival vehicle $v_{1}$, its parameter $\langle S, L, L, 1>$ represents that it will travel straightly with a lowest priority, no priority promotion, and is the first vehicle authorized to pass. Similarly, $v_{4}$ and $v_{6}$ are the second vehicle and the third one authorized to turn right at this intersection.

$b$. FAFP-SQ: Regard the whole intersection as a single S-CS; When the intersection is free, only choose and authorize the whole intersection to one vehicular platoon in which the earliest arrived vehicle is. As an example, Figure $6 \mathrm{~b}$ shows that $v_{1}, v_{2}$, and $v_{3}$ on the same lane are authorized as a platoon, and, $v_{2}$ will turn left while the other two will pass straightly.

c. FAFP-SQ-SV: Regard an intersection as a set of adjacent S-CSs; Each time, after choosing and authorizing a platoon with the manner same to FAFP-SQ, iteratively choose and authorize the earliest vehicle that is passable with those remnent-free S-CSs, from all left lanes. For the traffic situation in Figure $5, v_{1}, v_{2}$, and $v_{3}$ will be authorized as FAFP-SQ, but, because $\gamma_{2}$ is always not occupied, $v_{4}$ will be solely authorized to pass simultaneously, as shown in Figure 6c.

d. FAFP-MQ: Regard an intersection as a set of adjacent S-CSs; Each time, after choosing and authorizing a platoon with the manner same to FAFP-SQ, from the other vehicles and lanes, iteratively choose and authorize a platoon that includes the earliest arrival vehicle, and all members of which do not require any authorized S-CS. For example, the passable platoon on the same lane covers $v_{4}$ and $v_{5}$ as shown in Figure 6d. 


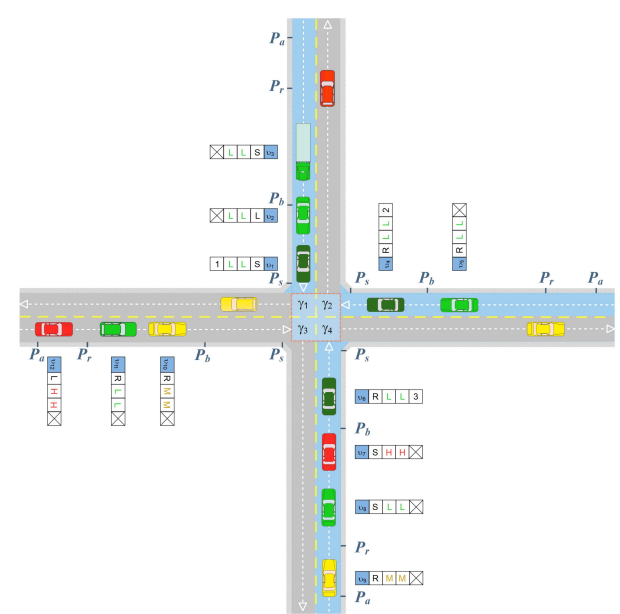

(a) FAFP-SV

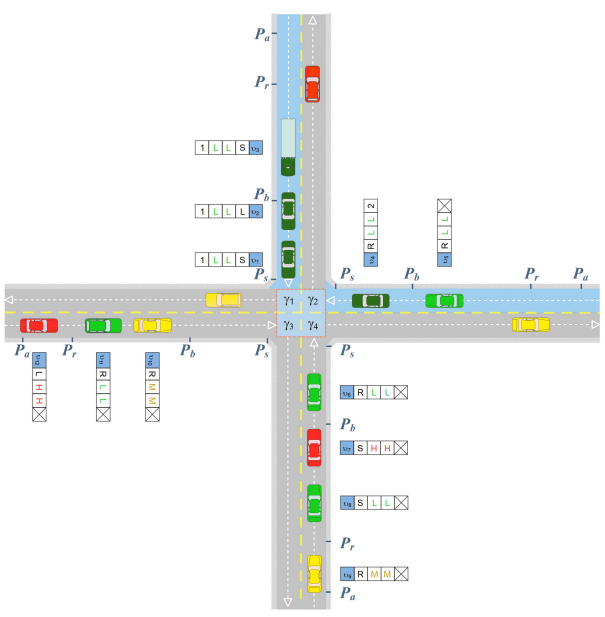

(c) FAFP-SQ-SV

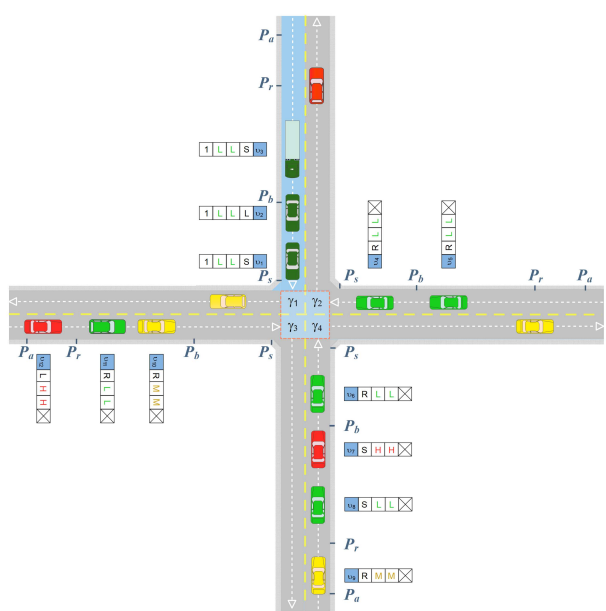

(b) FAFP-SQ

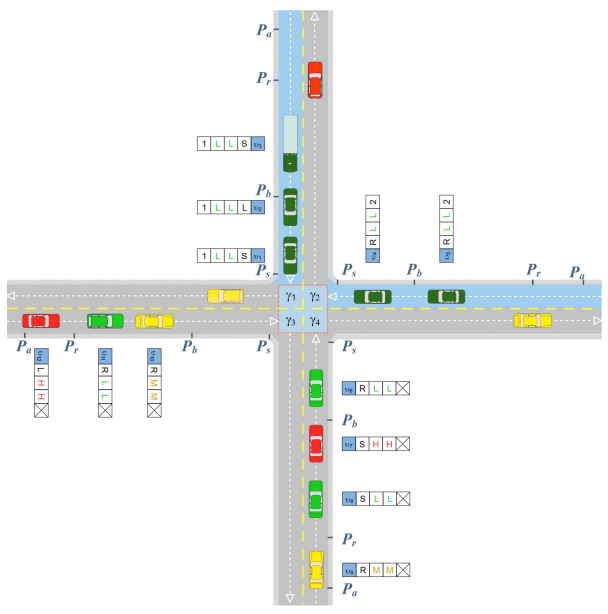

(d) FAFP-MQ

On this lane,existing authorized vehicle(s) _....... On this lane,no vehicle(s) authorized

Figure 6. Traffic Situations with Four FAFP-based Cooperation Policies. (a) FAFP-SV. (b) FAFP-SQ.

(c) FAFP-SQ-SV. (d) FAFP-MQ.

\section{(2) Four QoS-Oriented Policies}

a. HQEP-SV: Regard the whole intersection as a single S-CS; Processing all queues with GPI mechanism, and then choose the head vehicle with current highest priority (namely, Highest priority, or Medium priority when there is no vehicle $(\mathrm{H}))$ to authorize. For the cases that all head vehicles on different lanes own a same priority, this policy will finally degrade to be FAFP-SV policy. As shown in Figure $7 \mathrm{a}$, since $\gamma_{2}$ has been authorized to $v_{4}$ and $v_{4}$ will delay $v_{7}$, so the priority of $v_{4}$ and $v_{6}$ should be promoted to guarantee $v_{4}$ releasing obtained critical sections as soon as possible, and $v_{6}$ becomes a vehicle $(\mathrm{H})$;

b. HWFP-SQ: Regard the whole intersection as a single S-CS; When the intersection is free, select and authorize one platoon from the lane that owns a maximum weight value, as illustrated in Figure $7 \mathrm{~b}$. When all lanes have the same weight, this policy degrades to be a FAFP-SQ policy;

c. HWFP-SQ-SV: Regard an intersection as a set of adjacent S-CSs; Each time, after choosing and authorizing a platoon from the lane that has the maximum weight value, iteratively choose and authorize the vehicle with current highest priority from other lanes that does not require any authorized S-CS. The example is shown in Figure 7c. When all lanes have the same weight, this policy will degrade to be a FAFP-SQ-SV; 
d. HWFP-MQ: Regard an intersection as a set of adjacent S-CSs; Each time, after choosing and authorizing a platoon with the manner same to HWFP-SQ, from vehicles on other lanes, iteratively choose and authorize a platoon that owns the maximum weight, and all members of which do not require any authorized S-CS, such as that in Figure 7d. When all lanes own the same weight, this policy will be equal to FAFP-MQ.

As we have expected, the HQEP policy should have better performance and flexibility, and "-MQ" policies should have better parallelism degree. However, whether it conforms to our expectation still needs to verify. In the next section, we will compare all these different policies.

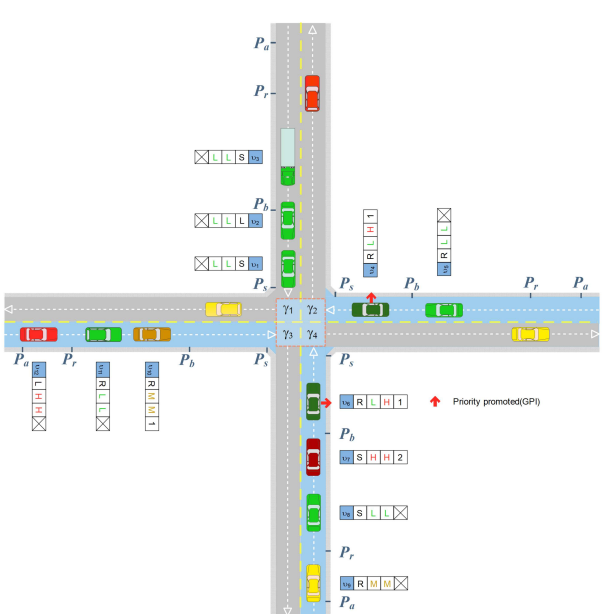

(a) HQEP-SV

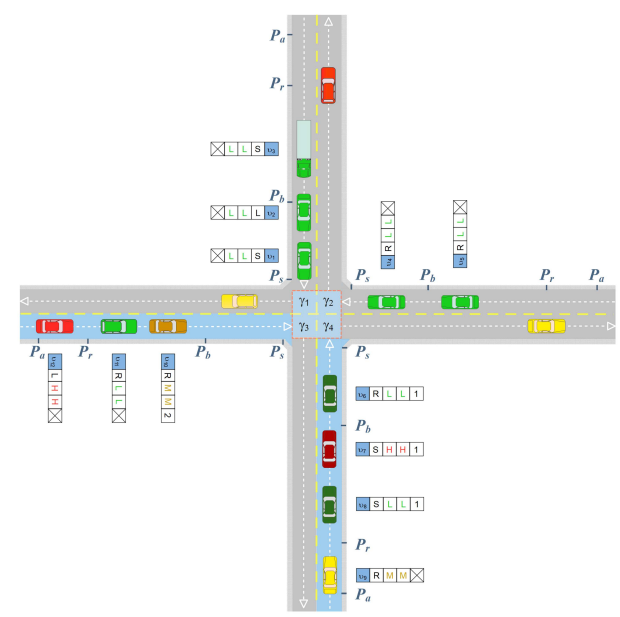

(c) HWFP-SQ-SV

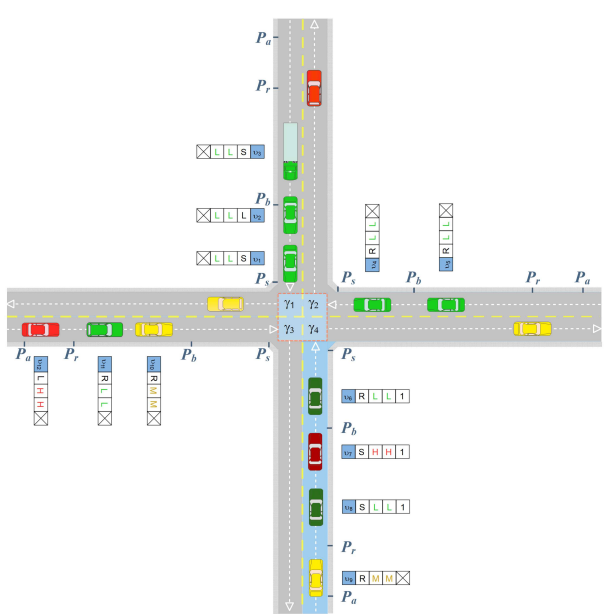

(b) HWFP-SQ

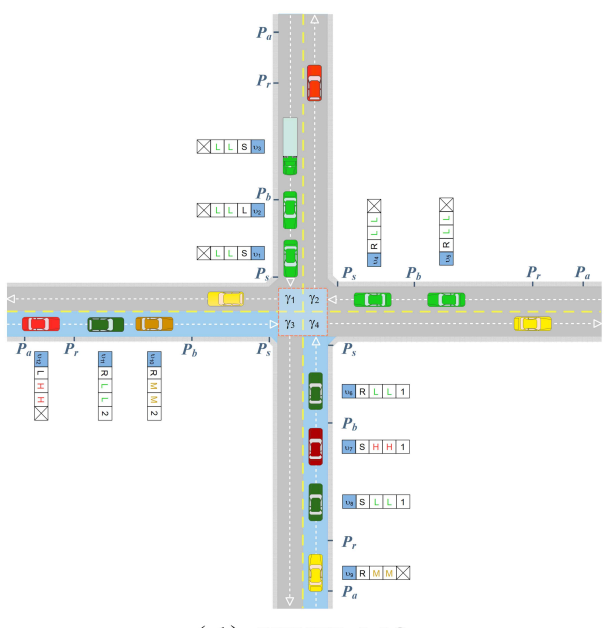

(d) HWFP-MQ

Figure 7. Traffic Situations with Four QoS-oriented Cooperation Policies. (a) HQEP-SV. (b) HWFP-SQ. (c) HWFP-SQ-SV. (d) HWFP-MQ.

\section{Verification Experiments and Analysis}

All models, policies, and mechanisms presented above have been finally implemented within a new version of a parameter-configurable traffic simulator QoS-CITS (v2.1), which is a testbed fully designed and developed by ourselves with C\# language on Windows platform, and where, several existing traffic models have been also adopted, especially the Ackermann steering model, Krauss car-following model. With this simulator, a plenty of experiments are conducted. 


\subsection{Parameter Settings}

To verify the function and performance of all these policies, we firstly construct a basic traffic environment, where one square intersection $\mathbb{I}$ is separated into four $3.5 \mathrm{~m} \times 3.5 \mathrm{~m} \mathrm{~S}$-CSs as shown in Figure 8. Then, we use the Poisson distribution model as shown in Formula (2) to control the density of traffic flow of each lane, where $\lambda$ and $t$ are the average arrival rate of the vehicles and the length of time interval, separately. In addition, in the following experiments, the variable $k$ is set to $1, t$ is not lower than $1 \mathrm{~s}$, and $\lambda$ is assigned a value within [0.01,1.0]. Table 2 shows the settings of parameters.

$$
P_{k}=\frac{(\lambda t)^{k} * e^{-\lambda t}}{k !}
$$

Table 2. Parameter Setting of QoS-CITS Simulator.

\begin{tabular}{cccccc}
\hline Parameter & Value (Scope) & Parameter & Value (Scope) & Parameter & Value (Scope) \\
\hline$\left|\zeta_{\gamma}^{\mathbb{I}}\right|$ & 4 & $\left|\zeta_{\ell} \mathrm{I}\right|$ & 4 & $\gamma \cdot l$ & $3.5 \mathrm{~m}$ \\
$\gamma . w$ & $3.5 \mathrm{~m}$ & $v . l$ & $3.5 \mathrm{~m}, 4.5 \mathrm{~m}$ & $v . w$ & $1.5 \mathrm{~m}, 1.8 \mathrm{~m}$ \\
$a_{a}$ & $4 \mathrm{~m} / \mathrm{s}^{2}$ & $-a_{d}$ & $-4 \mathrm{~m} / \mathrm{s}^{2}$ & $\Delta c$ & $2 \mathrm{~m}$ \\
$\Delta c^{\prime}$ & $0.5 \mathrm{~m}$ & $d_{a}$ & $8 \mathrm{~m} \sim 10.5 \mathrm{~m}$ & $d_{r}$ & $10 \mathrm{~m} \sim 70 \mathrm{~m}$ \\
$d_{b}$ & $6 \mathrm{~m}$ & $d_{\gamma}$ & $1.42 \mathrm{~m} \sim 20 \mathrm{~m}$ & $v_{m}$ & $10 \mathrm{~m} / \mathrm{s}$ \\
$v_{r}$ & $4 \mathrm{~m} / \mathrm{s} \sim 10 \mathrm{~m} / \mathrm{s}$ & $v_{\gamma}$ & $6 \mathrm{~m} / \mathrm{s} \sim 10 \mathrm{~m} / \mathrm{s}$ & $p_{v . l=5 m}$ & $1 \% \sim 100 \%$ \\
$p_{H}$ & $1 \% \sim 30 \%$ & $p_{M}$ & $1 \% \sim 40 \%$ & $p_{L}$ & $40 \% \sim 90 \%$ \\
$\lambda$ & $0.01 \sim 1.0$ & $\beta_{1}$ & $0 \sim 1.0$ & $\beta_{2}$ & $0 \sim 1.0$ \\
$\beta_{3}$ & $0 \sim 1.0$ & $\varphi_{H}$ & 100 & $\varphi_{M}$ & 10 \\
$\varphi_{L}$ & 1 & $N$ & $1 \sim 10$ & $\Delta p$ & $10 \mathrm{~ms}$ \\
\hline
\end{tabular}

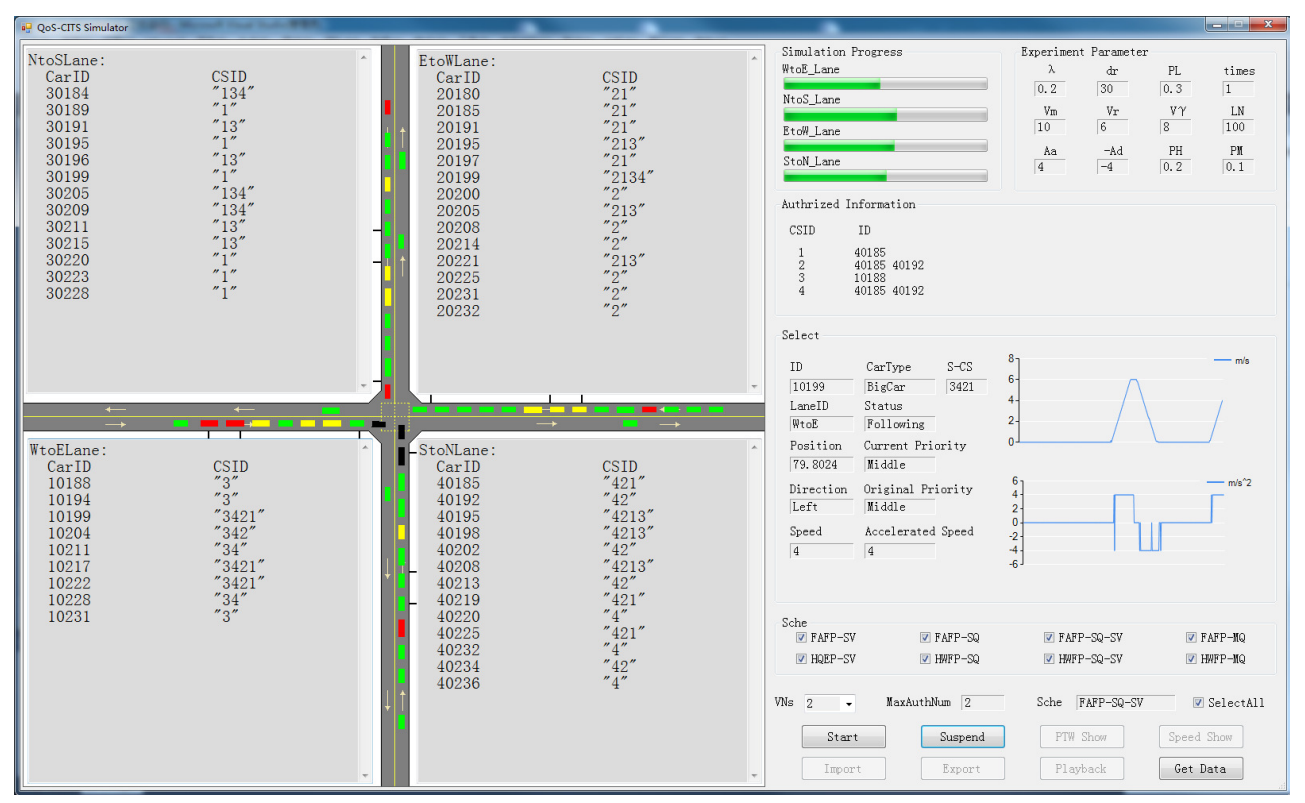

Figure 8. The Traffic Simulator QoS-CITS (v2.1).

\subsection{Experiments and Analysis}

Considering the key constraints to guarantee cooperation safety, QoS of vehicles $(\mathrm{H})$ (or vehicles (M) when there is no vehicle $(\mathrm{H})$ ), and the traffic throughput of one intersection, a series of experiments have been designed and carried out, and further, corresponding scheduling effects are analyzed as below. 


\section{(1) Promotion of Passing-Through Orders}

These designed policies are firstly verified under a same traffic situation, totally covering 200 vehicles in each experiment. Figures 9 and 10 shows the experimental results, where exist four vehicles (H): 20031 and 20046 on lane $\ell_{2}, 10201$ and 10211 on lane $\ell_{1}$. It can be observed that authorization orders of these four vehicles $(\mathrm{H})$ are promoted by these eight policies to be $\langle-2,6,-9,-8\rangle,\langle 1,3,0,0\rangle$, $\langle-1,2,3,6\rangle,\langle 0,2,0,2\rangle,\langle 6,12,12,12\rangle,\langle 10,11,14,14\rangle,\langle 9,8,12,13\rangle$, and $\langle 9,8,11,10\rangle$, respectively. It is clear that all four QoS-oriented policies authorize all vehicles $(\mathrm{H})$ to pass through preferentially, and those platoon-based policies also performed better. This experiment is carried out with a bigger $P_{H}$ value 0.2 , which means more vehicles $(\mathrm{H})$ will exist in traffic flows. However as is well known, such scenes are very scarcely in reality.
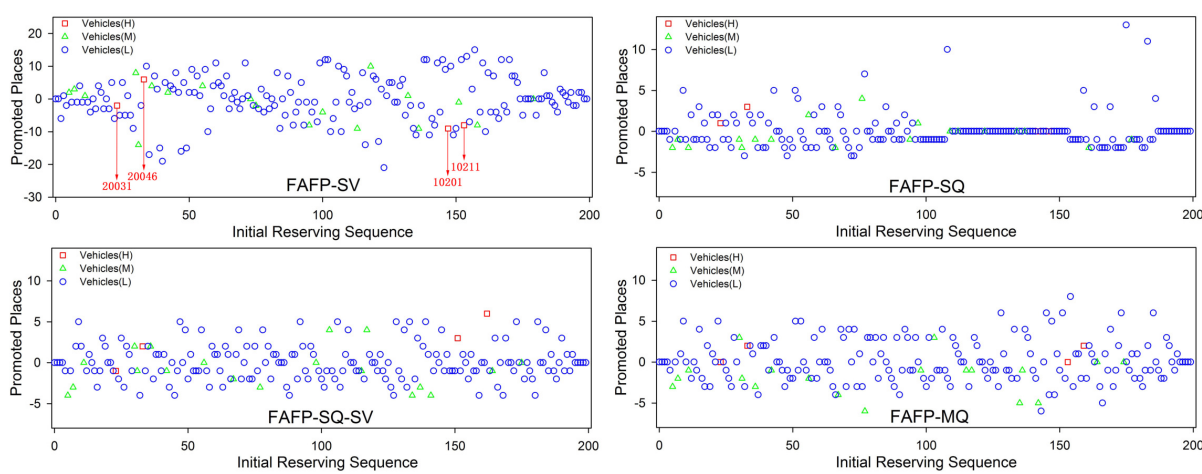

Figure 9. Comparison of Passing-through Orders of Four FAFP-based Policies: $\lambda=0.15, p_{v . l=4.5 m}=$ $30 \%, p_{H}=5 \%, p_{M}=10 \%, p_{L}=85 \%, d_{a}=8 \mathrm{~m}, d_{r}=30 \mathrm{~m}, v_{m}=10 \mathrm{~m} / \mathrm{s}, v_{r}=6 \mathrm{~m} / \mathrm{s}, v_{\gamma}=8 \mathrm{~m} / \mathrm{s}$, $N=2$.
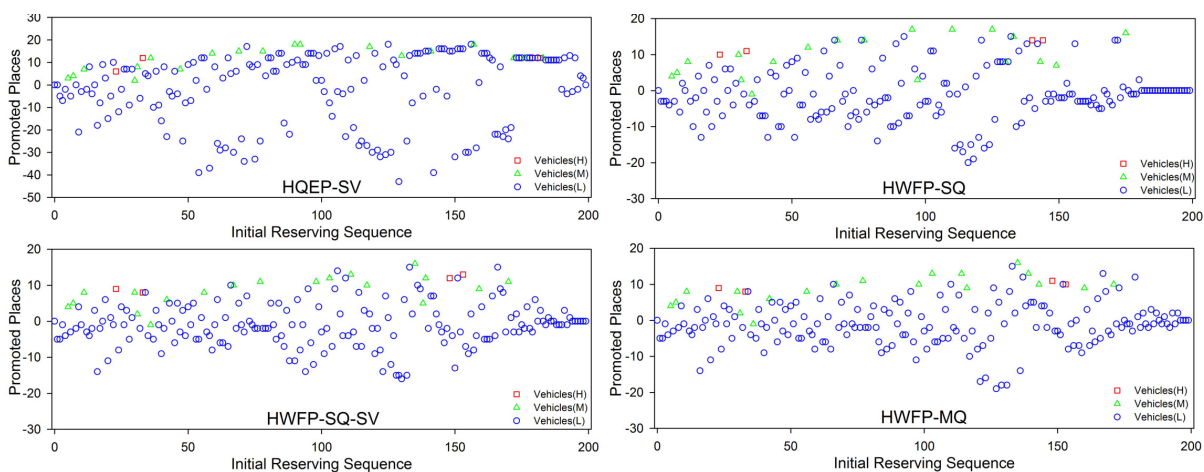

Figure 10. Comparison of Passing-through Orders of Four QoS-oriented Policies: $\lambda=0.15, p_{v . l=4.5 m}=$ $30 \%, p_{H}=5 \%, p_{M}=10 \%, p_{L}=85 \%, d_{a}=8 \mathrm{~m}, d_{r}=30 \mathrm{~m}, v_{m}=10 \mathrm{~m} / \mathrm{s}, v_{r}=6 \mathrm{~m} / \mathrm{s}, v_{\gamma}=8 \mathrm{~m} / \mathrm{s}$, $N=2$.

The relevant experimental results are shown in Figures 11 and 12, from which we can find these four QoS-oriented policies still perform better to guarantee the QoS of vehicles $(\mathrm{H})$. Significantly, HQEP-SV has exhibited the outstanding capability to authorize vehicles $(\mathrm{H})$ preferentially because the single-vehicle authorization and the GPI mechanism make it more flexible. It needs to be noted that the number of promoted places is not the unique factor to measure these policies. As aforementioned, there are still other important indicators need to be concerned, typically the average delay time of all vehicles $(\mathrm{H})$ and the throughput of any intersection. 

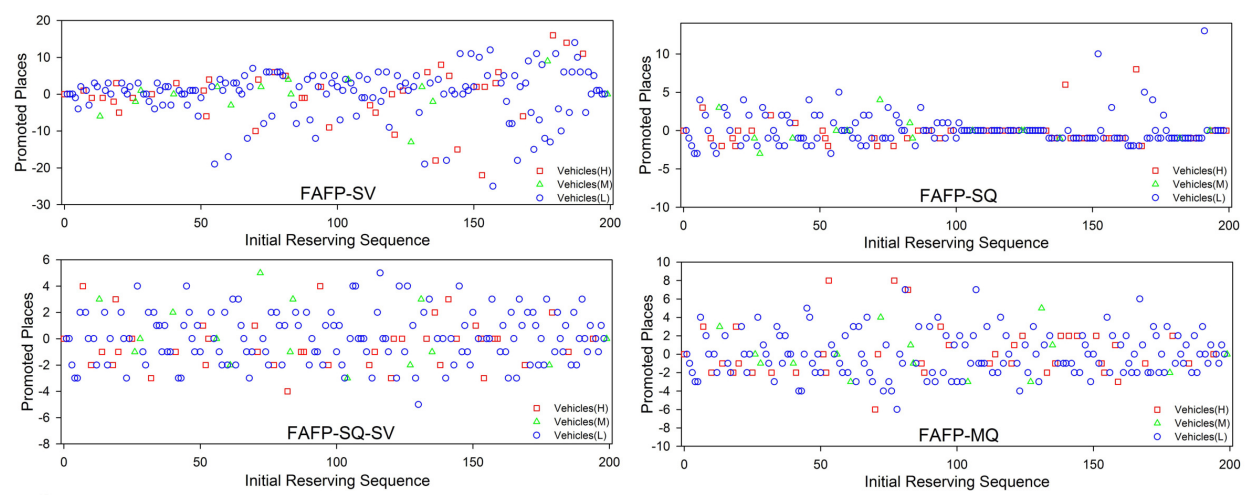

Figure 11. Comparison of Passing-through Orders of Four FAFP-based Policies: $\lambda=0.15, p_{v . l}=4.5 \mathrm{~m}=$ $30 \%, p_{H}=20 \%, p_{M}=10 \%, p_{L}=70 \%, d_{a}=8 \mathrm{~m}, d_{r}=30 \mathrm{~m}, v_{m}=10 \mathrm{~m} / \mathrm{s}, v_{r}=6 \mathrm{~m} / \mathrm{s}, v_{\gamma}=8 \mathrm{~m} / \mathrm{s}$, $N=2$.
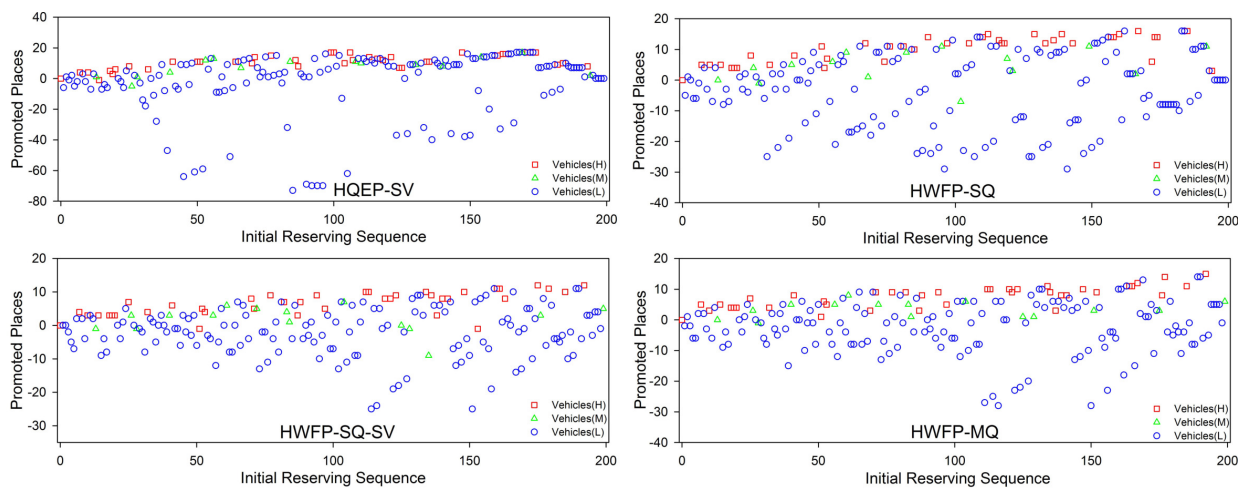

Figure 12. Comparison of Passing-through Orders of Four QoS-oriented Policies: $\lambda=0.15, p_{v . l=4.5 m}=$ $30 \%, p_{H}=20 \%, p_{M}=10 \%, p_{L}=70 \%, d_{a}=8 \mathrm{~m}, d_{r}=30 \mathrm{~m}, v_{m}=10 \mathrm{~m} / \mathrm{s}, v_{r}=6 \mathrm{~m} / \mathrm{s}, v_{\gamma}=8 \mathrm{~m} / \mathrm{s}$, $N=2$.

(2) Delay Time of Vehicles $\{\mathrm{H}, \mathrm{M}, \mathrm{L}\}$

Delay time is the real indicator that reflects how long one vehicle has been blocked. Although we have devoted to finding novel policies to authorize vehicles $(H)$ preferentially, a few vehicles $(H)$ are still delayed when there simultaneously exist vehicles $(\mathrm{H})$ on different lanes. This is a normal phenomenon in real transportation procedures. Thereout, we have drew up a statistics-based evaluation indicator, the average delay time, to appraise the effect of these policies. Concretely, for each experimental variable, a series of experiments have been conducted, and each time the traffic flows are composed of 200 vehicles.

\section{- $\quad$ Reservation Distance: $d_{r}$}

The influence of reservation distance $d_{r}$, corresponding to the position $P_{r}$, is the first experimental indicator. In our model, the value of $d_{r}$ will mainly affect the length of $Q_{\ell}$. The bigger the value of $d_{r}$ is appointed, the more vehicles are allowed to be buffered in $Q_{\ell}$. It implicitly means that one vehicle $(\mathrm{H})$ is able to be perceived earlier by II-Agent, thereby, can be authorized earlier.

Figures 13 and 14 shows experimental results when changing $d_{r}$ from $10 \mathrm{~m}$ to $70 \mathrm{~m}$. It can be observed that along with the increase of $d_{r}$ the average delay time of all vehicles rises, especially for FAFP-SV policy. That is mainly because that there are more vehicles buffered in these $Q_{\ell}$, and furthermore, leading to more vehicles delayed. However, it can be also observed that, all QoS-oriented policies can obviously decrease the average delay time of vehicles with higher priorities, 
meanwhile, be influenced very little by $d_{r}$. This demonstrates that these policies possess the capability to warrant QoS of vehicles with higher priorities. Furthermore, we can find that queue-based policies, covering FAFP-SQ-SV, FAFP-MQ, HWFP-SQ-SV, and HWFP-MQ, have also reduced the delay of vehicles with higher priorities significantly. Especially, HWFP-SQ-SV exhibited the best effect, which is because that the "SV" property allows vehicles on other lanes to obtain unauthorized S-CSs.
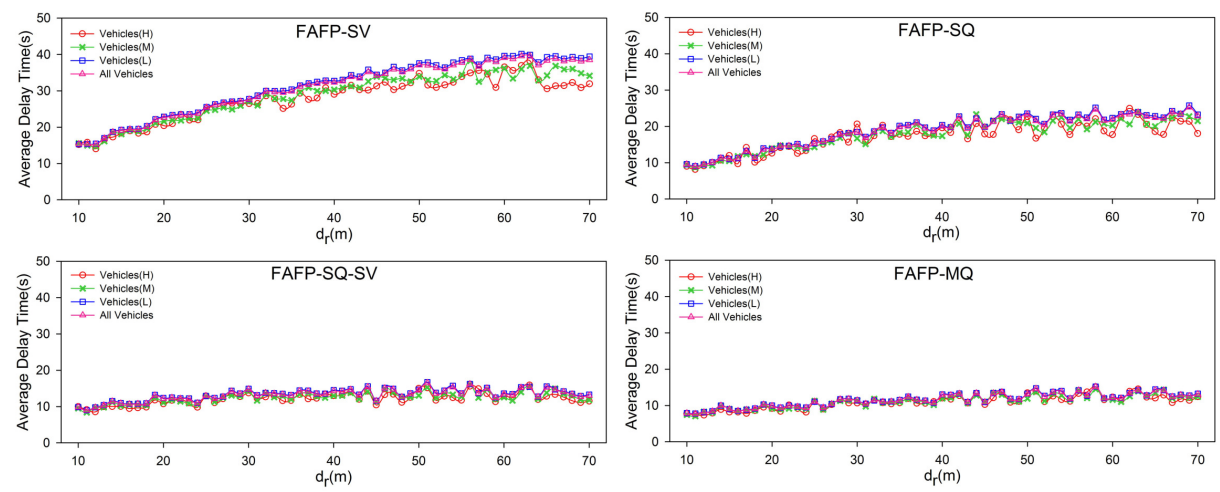

Figure 13. Average Delay Time of Vehicles $\{\mathrm{H}, \mathrm{M}, \mathrm{L}\}$ with the Change of $d_{r}$ of Four FAFP-based Policies: $\lambda=0.15, p_{v . l=4.5 m}=30 \%, p_{H}=5 \%, p_{M}=10 \%, p_{L}=85 \%, d_{a}=8 \mathrm{~m}, v_{m}=10 \mathrm{~m} / \mathrm{s}, v_{r}=6 \mathrm{~m} / \mathrm{s}$, $v_{\gamma}=8 \mathrm{~m} / \mathrm{s}, N=2$.
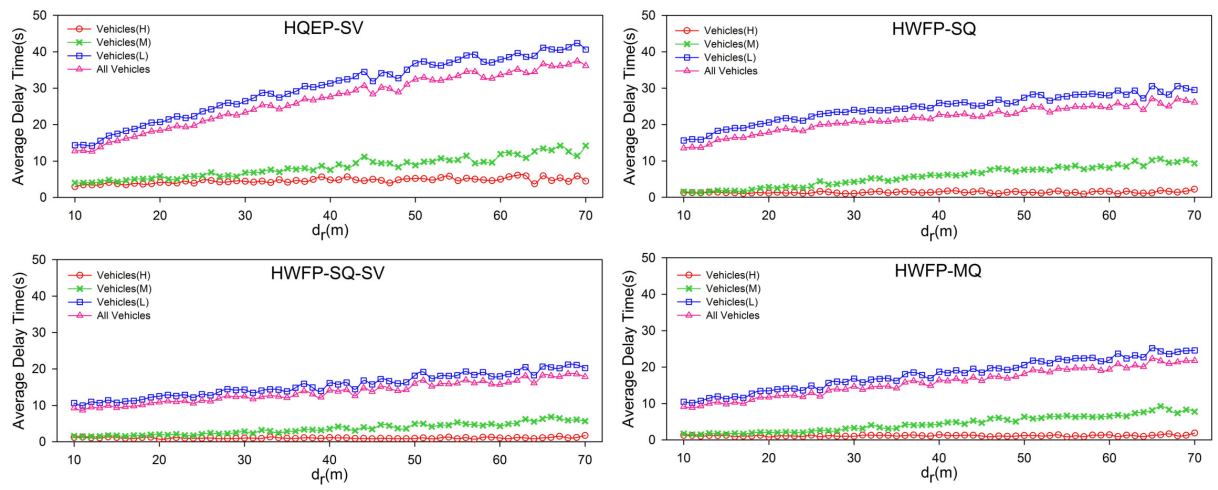

Figure 14. Average Delay Time of Vehicles $\{\mathrm{H}, \mathrm{M}, \mathrm{L}\}$ with the Change of $d_{r}$ of Four QoS-oriented Policies: $\lambda=0.15, p_{v . l=4.5 m}=30 \%, p_{H}=5 \%, p_{M}=10 \%, p_{L}=85 \%, d_{a}=8 \mathrm{~m}, v_{m}=10 \mathrm{~m} / \mathrm{s}, v_{r}=6 \mathrm{~m} / \mathrm{s}$, $v_{\gamma}=8 \mathrm{~m} / \mathrm{s}, N=2$.

- Density of Traffic Flow: $\lambda$

Traffic density is another vital indicator that maybe changes in different situation and affects the traffic efficiency. In our experiments, the influence of $\lambda$ to all policies is evaluated within the value scope $[0.01,1.0]$ and with normal proportion of vehicles $(\mathrm{H})$. After analyzing the results as shown in Figures 15 and 16, it can be concluded that QoS-oriented policies indeed exhibited good performance even though $\lambda$ exceeds the value 0.13 . However, from this point 0.13 , the average delay time of each FAFP-based policy increases jumpily. The main reason for this phenomenon is that when $P_{H}$ is small, there only exist very few vehicles $(\mathrm{H})$, thus, the phenomenon that one vehicle $(\mathrm{H})$ blocks vehicles $(\mathrm{H})$ on other lanes will rarely happen. That is to say, under normal traffic situations, $\lambda$ has very limited impact on the performance of these QoS-oriented policies. 

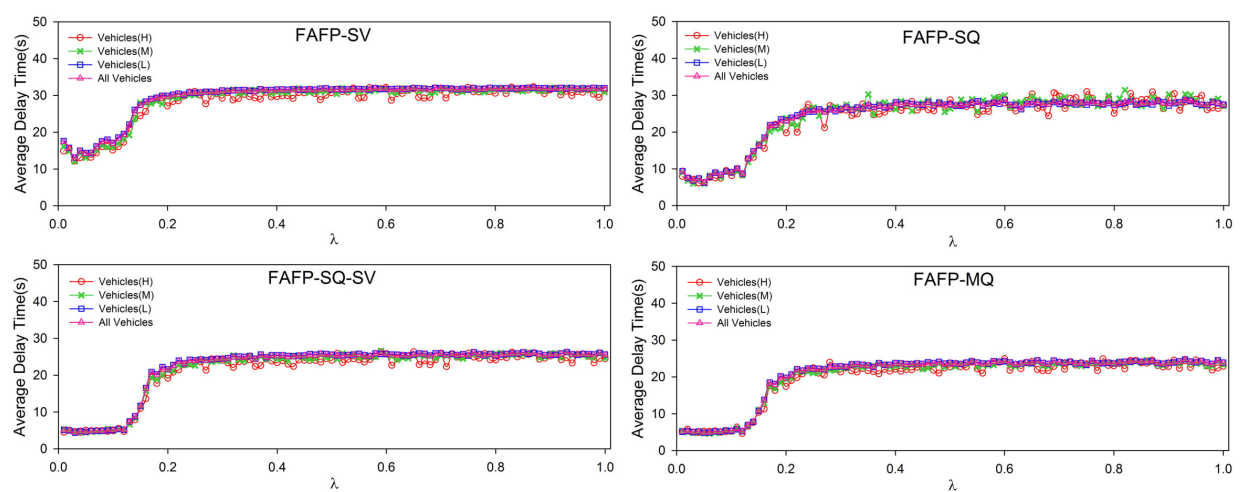

Figure 15. Average Delay Time of Vehicles with Different $\lambda$ of Four FAFP-based Policies : $p_{v . l=4.5 m}=$ $30 \%, p_{H}=5 \%, p_{M}=10 \%, p_{L}=85 \%, d_{a}=8 \mathrm{~m}, d_{r}=30 \mathrm{~m}, v_{m}=10 \mathrm{~m} / \mathrm{s}, v_{r}=6 \mathrm{~m} / \mathrm{s}, v_{\gamma}=8 \mathrm{~m} / \mathrm{s}$, $N=2$.
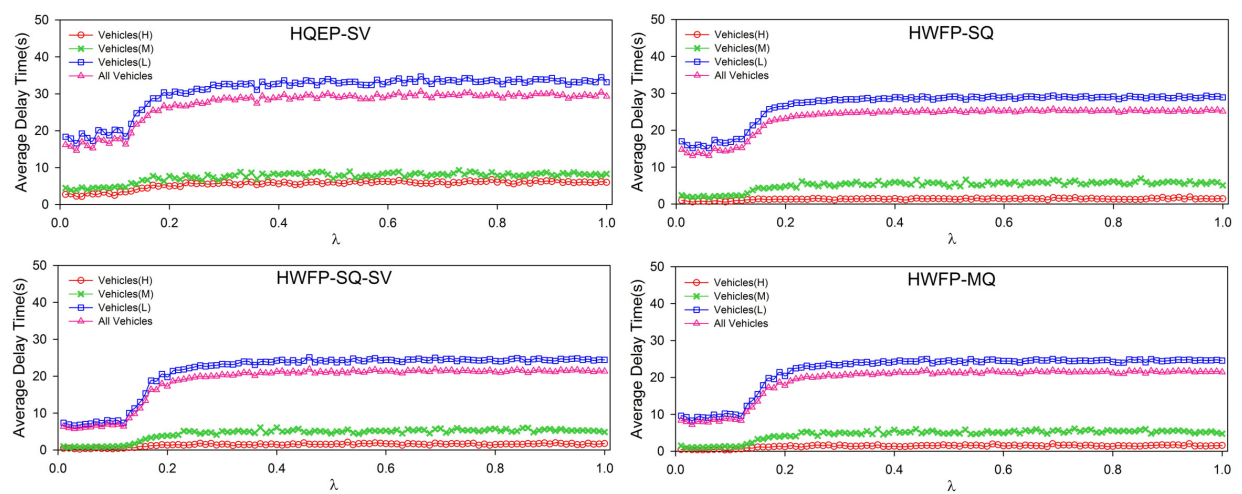

Figure 16. Average Delay Time of Vehicles with Different $\lambda$ of Four QoS-oriented Policies: $p_{v . l=4.5 m}=$ $30 \%, p_{H}=5 \%, p_{M}=10 \%, p_{L}=85 \%, d_{a}=8 \mathrm{~m}, d_{r}=30 \mathrm{~m}, v_{m}=10 \mathrm{~m} / \mathrm{s}, v_{r}=6 \mathrm{~m} / \mathrm{s}, v_{\gamma}=8 \mathrm{~m} / \mathrm{s}$, $N=2$.

- Proportion of Large Vehicles: $P_{v . l=4.5 m}$

From the models of intelligent vehicles and S-CS, we know that various vehicles with different sizes will occupy different amount of S-CSs when passing through an intersection, and the passingthrough time will be also discrepant. Particularly, when taking a left-turning maneuver, larger vehicles will maybe occupy all four S-CSs as shown in Figure 2a, while small vehicles will only enter into three of these S-CSs. Obviously, the latter cases will possibly allow the concurrency of multiple passing-through procedures. So, the influence of the proportion of lager vehicles also needs to be evaluated. Figures 17 and 18 presents a group of experimental results. It is clear that the average delay time of vehicles $(\mathrm{H})$ with queue-based policies still keep lower than that with FAFP-SV and HQEP-SV policies. It can be also concluded that all QoS-oriented policies are effective to guarantee the QoS of vehicles $(\mathrm{H})$ under situations with different $P_{v . l}$. 

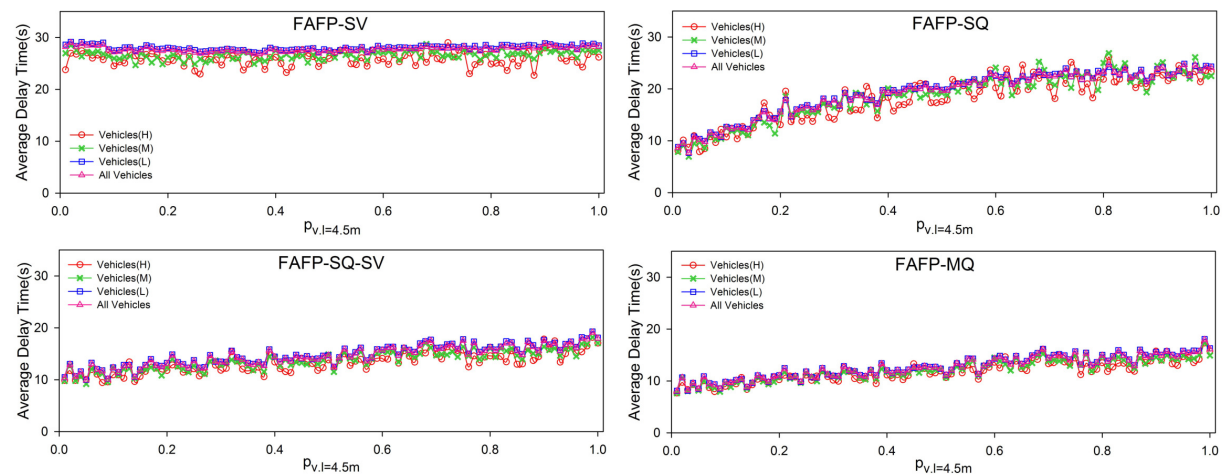

Figure 17. Average Delay Time of Vehicles with Different $P_{v . l=4.5 m}$ of Four FAFP-based Policies: $\lambda=0.15, p_{H}=5 \%, p_{M}=10 \%, p_{L}=85 \%, d_{a}=8 \mathrm{~m}, d_{r}=30 \mathrm{~m}, v_{m}=10 \mathrm{~m} / \mathrm{s}, v_{r}=6 \mathrm{~m} / \mathrm{s}, v_{\gamma}=8 \mathrm{~m} / \mathrm{s}$, $N=2$.
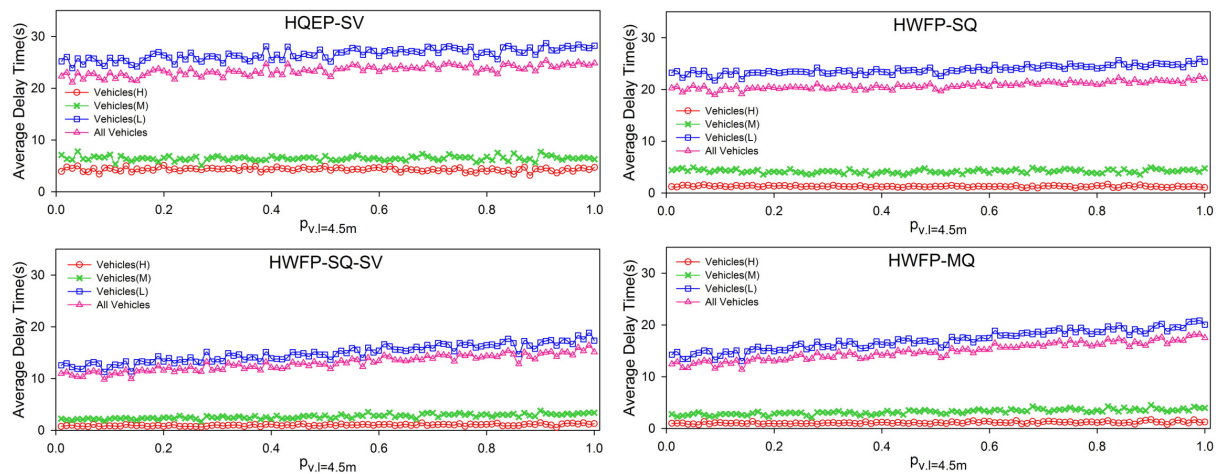

Figure 18. Average Delay Time of Vehicles with Different $P_{v . l=4.5 m}$ of Four QoS-oriented Policies: $\lambda=0.15, p_{H}=5 \%, p_{M}=10 \%, p_{L}=85 \%, d_{a}=8 \mathrm{~m}, d_{r}=30 \mathrm{~m}, v_{m}=10 \mathrm{~m} / \mathrm{s}, v_{r}=6 \mathrm{~m} / \mathrm{s}, v_{\gamma}=8 \mathrm{~m} / \mathrm{s}$, $N=2$.

- Proportion of Vehicles (H): $P_{H}$

Through experimental results as shown in Figures 19 and 20, it can be found that under normal traffic situations where $\lambda$ is smaller, all QoS-oriented policies perform better than FAFP-based policies. But these experiments have also proved that, along with the increase of $P_{H}$ and $\lambda$, the performance of QoS-oriented policies become more different. Figures 19 and 20 also presents the cooperation effects when $\lambda$ is 0.8 , namely under heavy traffic-jam situations. From this figure, it can be found that all queue-based QoS-oriented policies are still capable to perform well even though the average delay time is increased somewhat. Of course, the exception is that HQEP-SV begins to degrade significantly since $P_{H}$ exceeds 0.7 . This is mainly because when the $P_{H}$ and $\lambda$ are all big enough, GPI will promote more vehicles $\{\mathrm{M}, \mathrm{L}\}$ to the highest priority that will eliminate the differences of vehicular emergencies. Extremely, HQEP-SV will finally degrade to FAFP-SV. 

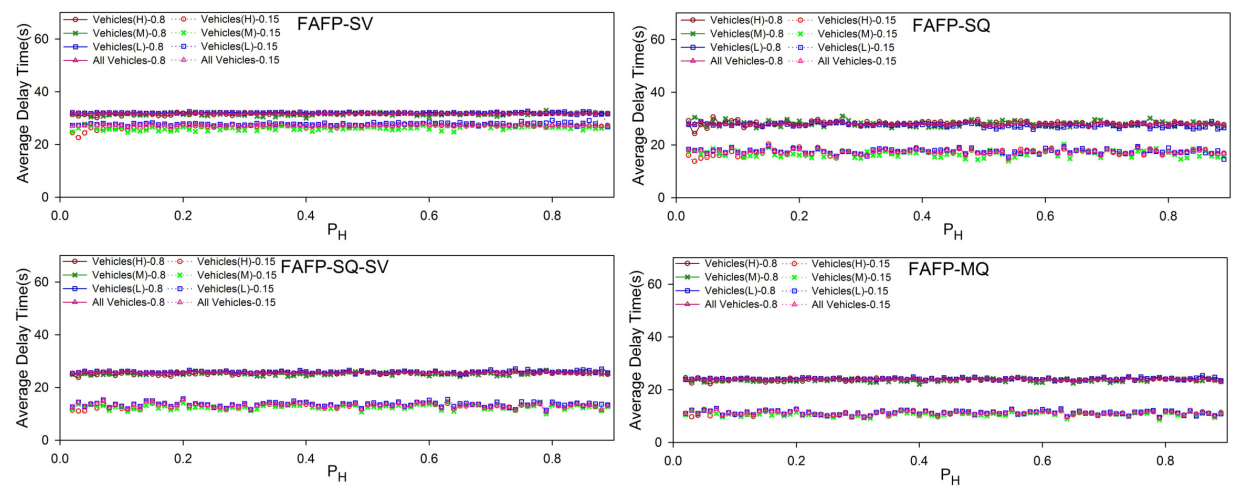

Figure 19. Average Delay Time of Vehicles with Different $P_{H}$ of Four FAFP-based Policies: $\lambda=$ $\{0.15,0.8\}, P_{v . l=4.5 m}=30 \%, p_{M}=10 \%, d_{a}=8 \mathrm{~m}, d_{r}=30 \mathrm{~m}, v_{m}=10 \mathrm{~m} / \mathrm{s}, v_{r}=6 \mathrm{~m} / \mathrm{s}, v_{\gamma}=8 \mathrm{~m} / \mathrm{s}$, $N=2$.
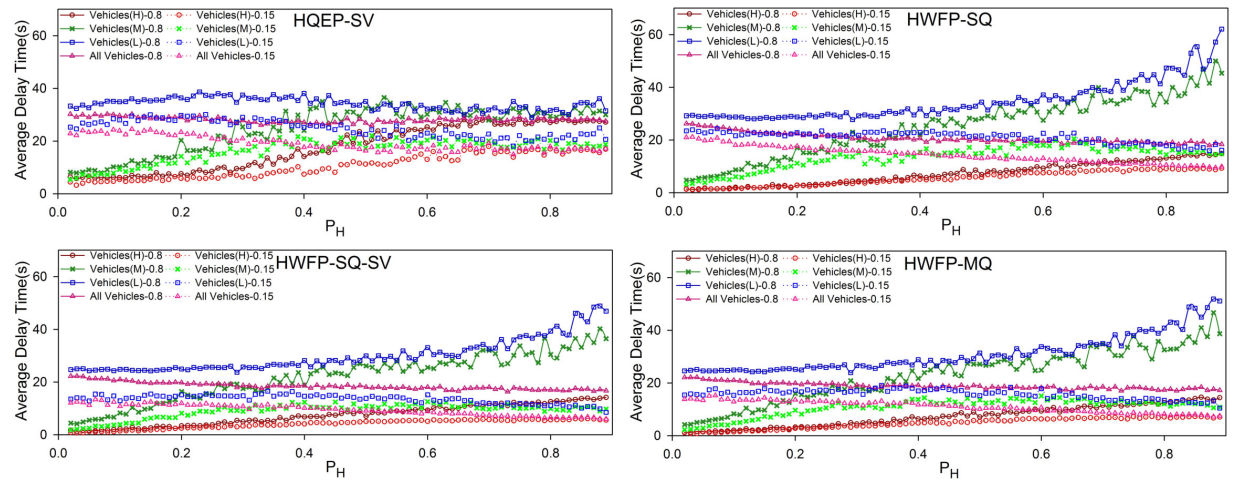

Figure 20. Average Delay Time of Vehicles with Different $P_{H}$ of Four QoS-oriented Policies: $\lambda=$ $\{0.15,0.8\}, P_{v . l=4.5 m}=30 \%, p_{M}=10 \%, d_{a}=8 \mathrm{~m}, d_{r}=30 \mathrm{~m}, v_{m}=10 \mathrm{~m} / \mathrm{s}, v_{r}=6 \mathrm{~m} / \mathrm{s}, v_{\gamma}=8 \mathrm{~m} / \mathrm{s}$, $N=2$.

\section{- Platoon Length: $N$}

Platoon length is in fact the amount of vehicles to be authorized each time. From the common sense, longer one platoon is, shorter the average delay time will be caused. But, it can be also imagined that a bigger platoon will maybe block vehicles on other lanes for a longer time. To find the influence of platoon length, presented as the variable $N$ within the value scope $[1,10]$, we conduct a series of experiments. Figures 21 and 22 presents the effects under normal traffic situations. From these results, it can be observed that when $N$ is greater than 1, namely authorizing a vehicular platoon at each time, the average delay time of each queue-based policy decreases obviously, meanwhile, QoS-oriented policies still warrant QoS of vehicles $(\mathrm{H})$ well. Since a flexible value of $N$ has been employed in queue-based QoS-oriented policies, as described in Algorithms 3 and 4, any vehicle $(\mathrm{H})$ could be authorized in time so long as it has submitted a reservation message. It is also very clear that, when $N$ is bigger than 4 , the average delay time caused by each QoS-oriented policy will level off. Of course, this effect is also relative to some other factors, such as $d_{r}, P_{H}, P_{v \cdot l}$, and $\lambda$. 

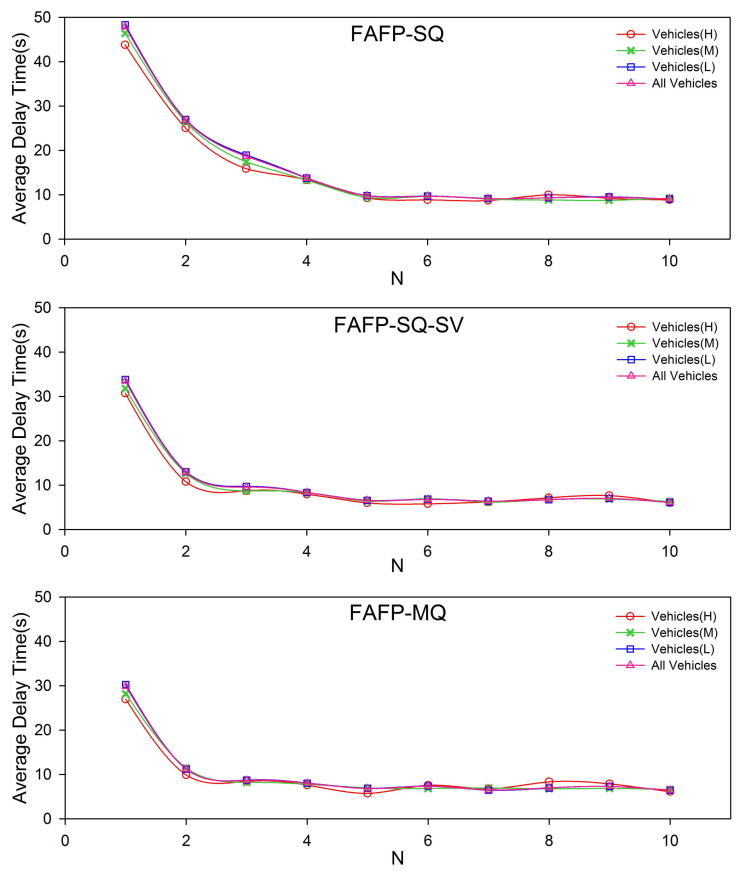

Figure 21. Average Delay Time of Vehicles with Different $N$ of Three FAFP-based Policies: $\lambda=0.15$, $P_{v . l=4.5 \mathrm{~m}}=30 \%, p_{H}=5 \%, p_{M}=10 \%, p_{L}=85 \%, d_{a}=8 \mathrm{~m}, d_{r}=30 \mathrm{~m}, v_{m}=10 \mathrm{~m} / \mathrm{s}, v_{r}=6 \mathrm{~m} / \mathrm{s}$, $v_{\gamma}=8 \mathrm{~m} / \mathrm{s}$.
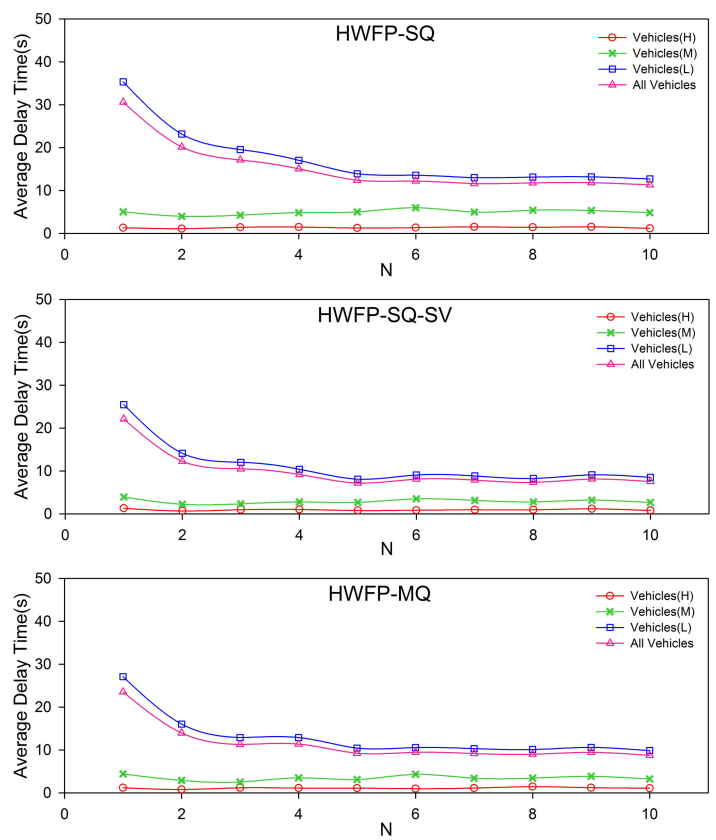

Figure 22. Average Delay Time of Vehicles with Different $N$ of Three QoS-oriented Policies: $\lambda=0.15$, $P_{v . l=4.5 m}=30 \%, p_{H}=5 \%, p_{M}=10 \%, p_{L}=85 \%, d_{a}=8 \mathrm{~m}, d_{r}=30 \mathrm{~m}, v_{m}=10 \mathrm{~m} / \mathrm{s}, v_{r}=6 \mathrm{~m} / \mathrm{s}$, $v_{\gamma}=8 \mathrm{~m} / \mathrm{s}$.

\section{(3) Analysis of Throughput at One Intersection}

Keeping high throughput is another important goal for the optimizing of traffic management. During our work, the throughput has been taken into account as well as the main target to authorize vehicles $(\mathrm{H})$ preferentially. While considering the QoS efficiency of these policies, the traffic throughput 
at an intersection is further evaluated statistically. Figure 23 shows the statistical results of eight policies aforementioned. From this figure, it can be observed that queue-based policies can improve the throughput prominently, and HQEP-SV exhibited better performance to authorize vehicles $(\mathrm{H})$ as early as possible. In addition, those queue-based policies, especially HWFP-SQ-SV and HWFP-MQ, can guarantee higher throughput because of their concurrency characteristic. Correspondingly, Figures 24-28 present the average delay along with the changing of variables $d_{r}, \lambda, P_{v . l}, N$, and $P_{H}$ with two typical values of $\lambda$, respectively. In addition, Figure 27 also indicates that HWFP-SQ-SV and HWFP-MQ always perform better than the other policies under both normal traffic situations and traffic-jam conditions.

As a whole, all these experimental results have demonstrated the performance of these policies from different aspects. It can be concluded that, both the queue-based and QoS-oriented properties are efficient to optimize the management of service-oriented traffic flows of intelligent vehicles.

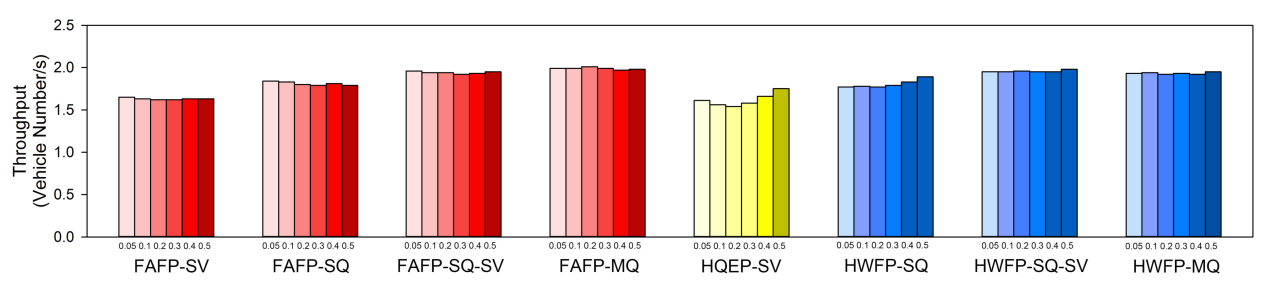

Figure 23. Comparison of Intersection Throughput: $\lambda=0.15, p_{v . l=4.5 m}=30 \%, p_{H}=$ $\{5 \%, 10 \%, 20 \%, 30 \%, 40 \%, 50 \%\}, p_{M}=10 \%, d_{a}=8 \mathrm{~m}, d_{r}=30 \mathrm{~m}, v_{m}=10 \mathrm{~m} / \mathrm{s}, v_{r}=6 \mathrm{~m} / \mathrm{s}$, $v_{\gamma}=8 \mathrm{~m} / \mathrm{s}, N=2$.
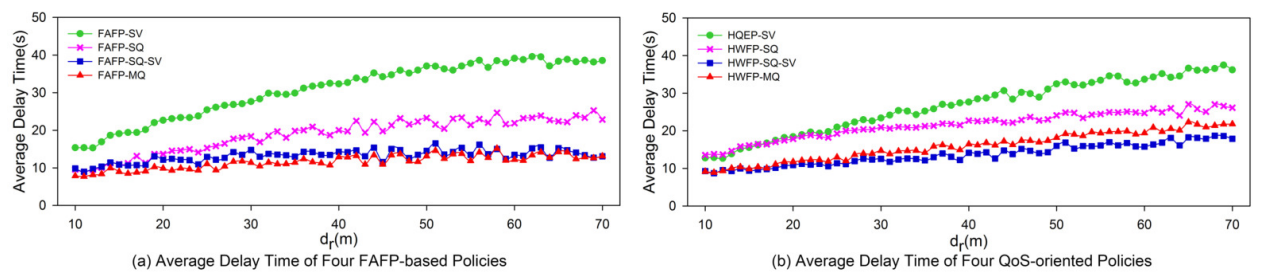

Figure 24. Average Delay Time of All Policies with Different $d_{r}: \lambda=0.15, p_{v . l=4.5 m}=30 \%, p_{H}=5 \%$, $p_{M}=10 \%, p_{L}=85 \%, d_{a}=8 \mathrm{~m}, v_{m}=10 \mathrm{~m} / \mathrm{s}, v_{r}=6 \mathrm{~m} / \mathrm{s}, v_{\gamma}=8 \mathrm{~m} / \mathrm{s}, N=2$.
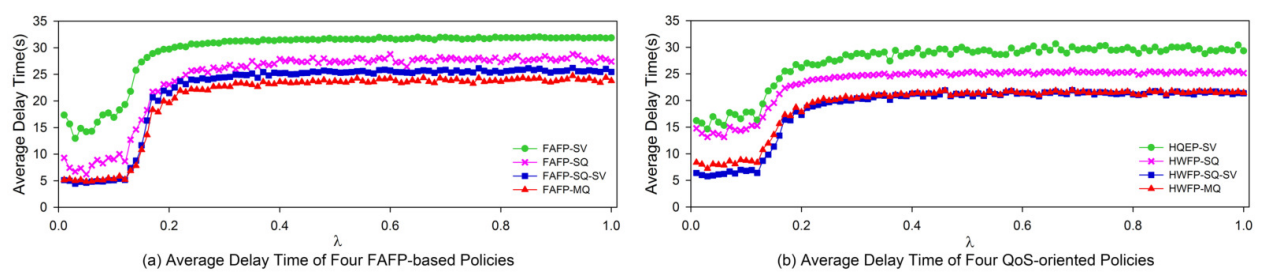

Figure 25. Average Delay Time of All Policies with Different $\lambda: p_{v . l=4.5 m}=30 \%, p_{H}=5 \%, p_{M}=10 \%$, $p_{L}=85 \%, d_{a}=8 \mathrm{~m}, d_{r}=30 \mathrm{~m}, v_{m}=10 \mathrm{~m} / \mathrm{s}, v_{r}=6 \mathrm{~m} / \mathrm{s}, v_{\gamma}=8 \mathrm{~m} / \mathrm{s}, N=2$.
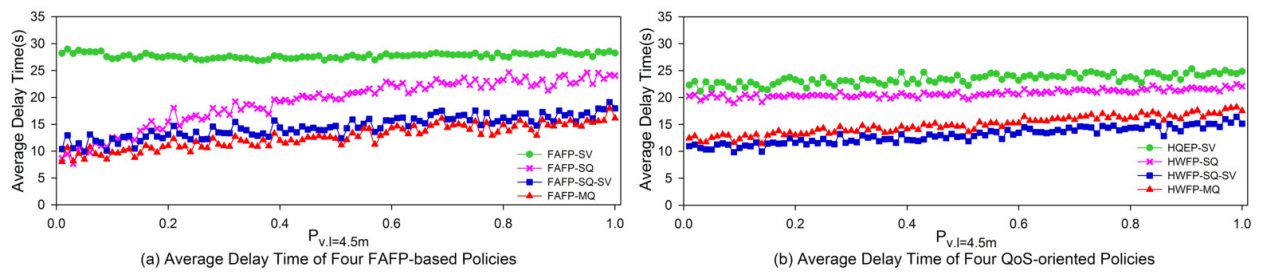

Figure 26. Average Delay Time of All Policies with Different $P_{v . l=4.5 m}: \lambda=0.15, p_{H}=5 \%, p_{M}=10 \%$, $p_{L}=85 \%, d_{a}=8 \mathrm{~m}, d_{r}=30 \mathrm{~m}, v_{m}=10 \mathrm{~m} / \mathrm{s}, v_{r}=6 \mathrm{~m} / \mathrm{s}, v_{\gamma}=8 \mathrm{~m} / \mathrm{s}, N=2$. 

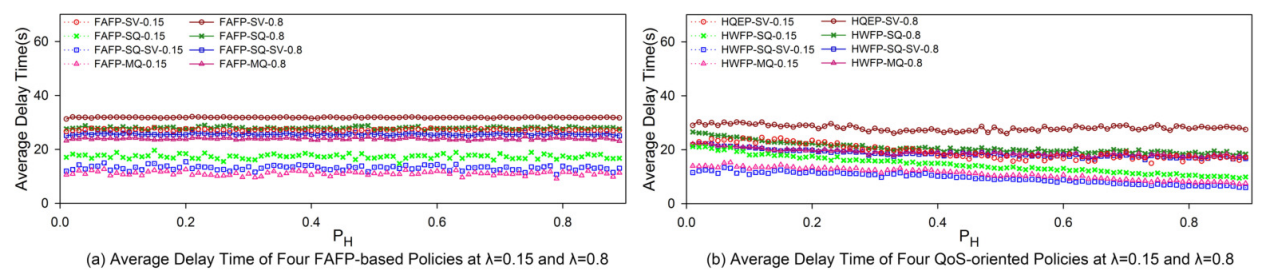

Figure 27. Average Delay Time of All Policies with Different $P_{H}: \lambda=\{0.15,0.8\}, P_{v . l=4.5 m}=30 \%$, $p_{M}=10 \%, d_{a}=8 \mathrm{~m}, d_{r}=30 \mathrm{~m}, v_{m}=10 \mathrm{~m} / \mathrm{s}, v_{r}=6 \mathrm{~m} / \mathrm{s}, v_{\gamma}=8 \mathrm{~m} / \mathrm{s}, N=2$.
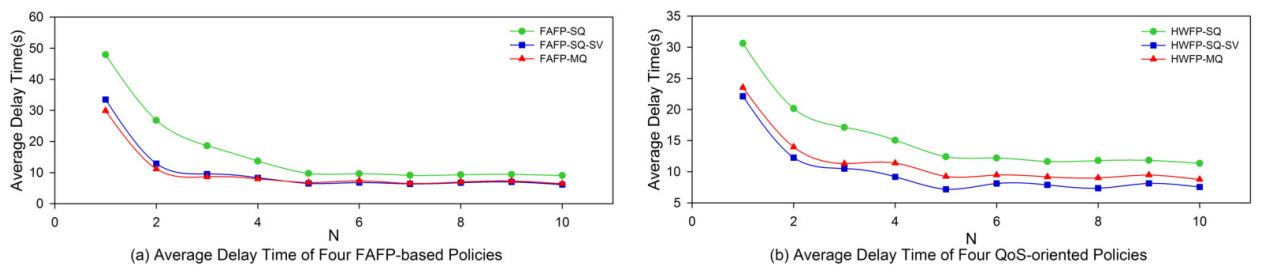

Figure 28. Average Delay Time of All Queue-based Policies with Different $N: \lambda=0.15, P_{v . l=4.5 m}=30 \%$, $p_{H}=5 \%, p_{M}=10 \%, p_{L}=85 \%, d_{a}=8 \mathrm{~m}, d_{r}=30 \mathrm{~m}, v_{m}=10 \mathrm{~m} / \mathrm{s}, v_{r}=6 \mathrm{~m} / \mathrm{s}, v_{\gamma}=8 \mathrm{~m} / \mathrm{s}$.

\section{Conclusions}

As a continuation of our previous study, a set of cooperation polices relative to QoS-oriented PTI problem are studied deeply in this paper. In particular, after analyzing the QoS features of vehicles $\{\mathrm{H}, \mathrm{M}\}$ during PTIs, several novel queue-based or/and platoon-based QoS-oriented policies are proposed and verified within our self-developed simulator QoS-CITS (v2.1). The large amount of experimental data have demonstrated that QoS-oriented policies can generally guarantee the traffic QoS of emergent vehicles better, namely, the delay of vehicles $(\mathrm{H})$ (or vehicles $(\mathrm{M})$ when there's no vehicle $(\mathrm{H})$ ) are decreased significantly. Meanwhile, all queue-based policies improve the throughput of intersection markedly. From this deeper study, we can conclude that queue-based QoS-oriented policies can guarantee both the traffic QoS of emergent vehicles and the traffic throughput. In particular, HWFP-SQ-SV and HWFP-MQ policies show better performances, which means that these policies are most suitable for the C-ITS in a city.

Our ongoing and future studies on this topic have been extending to the scope of road networks in a city domain, especially focusing on the prediction models and mechanisms of traffic flows in a city domain, and the QoS-oriented cooperative path-planning via traffic clouds and big data, and also the construction of an indoor Vehicle-In-Loop test bed, continuously perfecting our simulator.

Author Contributions: Conceptualization, K.Z. and A.d.L.F. and W.Z.; Methodology, K.Z. and M.W. and C.X.; Software, C.X. and M.W.; Validation, M.W. and Y.W.; Formal Analysis, K.Z. and C.X.; Investigation, K.Z.; Resources, K.Z. and Z.D.; Data Curation, M.W.; Writing-Original Draft Preparation, K.Z.; Writing-Review \& Editing, K.Z. and Y.W.; Visualization, C.X. and Y.W.; Supervision, K.Z.; Project Administration, K.Z.; Funding Acquisition, K.Z. and A.d.L.F.

Funding: This research was funded by [the Natural Science Foundation of China] grant number [61572403, 61502394], the Fundamental Research Funds for the Central Universities grant number [3102017zy022], the International Chair Drive for All.

Conflicts of Interest: The authors declare no conflict of interest. The founding sponsors had no role in the design of the study; in the collection, analyses, or interpretation of data; in the writing of the manuscript, and in the decision to publish the results. 


\section{References}

1. De La Fortelle, A.; Qian, X.; Diemer, S.; Grégoire, J.; Moutarde, F.; Bonnabel, S. Network of Automated Vehicles: The Autonet2030 Vision. In Proceedings of the 21st World Congress on the ITS World Congress (ITSWC), Detroit, MI, USA, 7-11 September 2014; Curran Associates, Inc.: Red Hook, NY, USA, 2014; pp. 2618-2626.

2. Ido, K.; Nadav, L.; Eran, B. An agent-based model of the emergence of cooperation and a fair and stable system optimum using ATIS on a simple road network. Transp. Res. Part C Emerg. Technol. 2017, 86, $183-201$.

3. Yang, L.Q.; Wang, F.Y. Driving into Intelligent Spaces with Pervasive Communications. IEEE Intell. Syst. 2007, 22, 12-15. [CrossRef]

4. Huang, S.; Sadek, A.W.; Zhao, Y.J. Assessing the Mobility and Environmental Benefits of Reservation-Based Intelligent Intersections Using an Integrated Simulator. IEEE Trans. Intell. Transp. Syst. 2012, 13, 1201-1214. [CrossRef]

5. Zhang, K.; Yang, A.; Su, H.; de La Fortelle, A.; Wu, X. Unified Modeling and Design of Reservation-based Cooperation Mechanisms for Intelligent Vehicles. In Proceedings of the 19th IEEE International Conference on Intelligent Transportation Systems(ITSC), Rio de Janeiro, Brazil, 1-4 November 2016; pp. 1192-1199.

6. Bujari, A.; Palazzi, C.E. Wireless Vehicular Networks for Car Collision Avoidance, 1st ed.; Springer: New York, NY, USA, 2013.

7. Zhang, K.; Zhang, D.; de La Fortelle, A.; Wu, X.; Grégoire, J. State-Driven Priority Scheduling Mechanisms for Driverless Vehicles Approaching Intersections. IEEE Trans. Intell. Transp. Syst. 2015, 16, 2487-2500. [CrossRef]

8. Li, L.; Wang, F.Y. Cooperative Driving at Blind Crossings using Intervehicle Communication. IEEE Trans. Veh. Technol. 2006, 55, 1712-1724. [CrossRef]

9. Levin, M.; Boyles, S.; Patel, R. Paradoxes of reservation-based intersection controls in traffic networks. Transp. Res. Part A Policy Pract. 2016, 90, 14-25. [CrossRef]

10. De La Fortelle, A. Analysis of Reservation Algorithms for Cooperative Planning at Intersections. In Proceedings of the 13th IEEE Conference on Intelligent Transportation Systems (ITSC), Funchal, Portugal, 19-22 September 2010; pp. 445-449.

11. Dresner, K.; Stone, P. A Multiagent Approach to Autonomous Intersection Management. Int. J. Artif. Intell. Res. 2008, 31, 591-656. [CrossRef]

12. Hausknecht, M.; Au, T.; Stone, P. Autonomous Intersection Management: Multi-intersection optimization. In Proceedings of the IEEE/RSJ 13th International Conference on Intelligent Robots and Systems (IROS), San Francisco, CA, USA, 25-30 September 2011; pp. 4581-4586.

13. Wuthishuwong, C.; Traechtler, A. Vehicle to Infrastructure Based Safe Trajectory Planning for Autonomous Intersection Management. In Proceedings of the IEEE 13th International Conference on ITS Telecommunications (ITST), Tampere, Finland, 5-7 November 2013; pp. 175-180.

14. Qian, X.; Altché, F.; Grégoire, J.; de La Fortelle, A. Autonomous Intersection Management Systems: Criteria, Implementation and Evaluation. IEEE Trans. Intell. Transp. Syst. 2017, 11, 182-189. [CrossRef]

15. Zhang, K.; Yang, A.; Su, H.; de La Fortelle, A.; Miao, K.; Yao, Y. Service-Oriented Cooperation Models and Mechanisms for Heterogeneous Driverless Vehicles at Continuous Static Critical Sections. IEEE Trans. Intell. Transp. Syst. 2017, 18, 1867-1881. [CrossRef]

16. Zhang, K.; Wang, M.; Su, H.; Yang, A.; de La Fortelle, A.; Miao, K.J. QoS-CITS: A Simulator for Serviceoriented Cooperative ITS of Intelligent Vehicles. In Proceedings of the 16th IEEE International Conference on Computer and Information Science (ICIS), Wuhan, China, 24-26 May 2017; pp. 1-6.

17. Varga, R.; Costea, A.; Florea, H.; Giosan, I.; Nedevschi, S. Super-sensor for 360-degree environment perception: Point cloud segmentation using image features. In Proceedings of the 2017 IEEE 20th International Conference on Intelligent Transportation Systems (ITSC), Yokohama, Japan, 16-19 October 2017; pp. 1-8.

18. Tang, X.; Gao, F.; Xu, G.; Ding, N.; Cai, Y.; Ma, M.; Liu, J. Sensor Systems for Vehicle Environment Perception in a Highway Intelligent Space System. Sensors 2014, 14, 8513-8527. [CrossRef] [PubMed] 
19. Rakotovao, T.; Puschini, D.; Mottin, J.; Rummelhard, L.; Negre, A.; Laugier, C. Intelligent Vehicle Perception: Toward the Integration on Embedded Many-core. In Proceedings of the 6th Workshop on Parallel Programming and Run-Time Management Techniques for Many-Core Architectures (PARMA-DITAM), Amsterdam, The Netherlands, 19-21 January 2015; pp. 7-12.

20. Lee, H.; Song, S.; Jo, S. 3D Reconstruction using a Sparse Laser Scanner and A Single Camera for Outdoor Autonomous Vehicle. In Proceedings of the 2016 IEEE 19th International Conference on Intelligent Transportation Systems (ITSC), Rio de Janeiro, Brazil, 1-4 November 2016; pp. 629-634.

21. Vivacqua, R.; Vassallo, R.; Martins, F. A Low Cost Sensors Approach for Accurate Vehicle Localization and Autonomous Driving Application. Sensors 2017, 17, 2359. [CrossRef] [PubMed]

22. Chen, C.; Seff, A.; Kornhauser, A.; Xiao, J. DeepDriving: Learning Affordance for Direct Perception in Autonomous Driving. In Proceedings of the 2015 IEEE International Conference on Computer Vision (ICCV), Santiago, Chile, 7-13 December 2015; pp. 2722-2730.

23. Qiu, H.; Ahmad, F.; Govindan, R.; Gruteser, M.; Bai, F.; Kar, G. Augmented Vehicular Reality: Enabling Extended Vision for Future Vehicles. In Proceedings of the 18th International Workshop on Mobile Computing Systems and Application, Sonoma, CA, USA, 21-22 February 2017; pp. 67-72.

24. Abualhoul, M.; Merdrignac, P.; Shagdar, O.; Nashashibi, F. Study and Evaluation of Laser-based Perception and Light Communication for A Platoon of Autonomous Vehicles. In Proceedings of the 2016 IEEE 19th International Conference on Intelligent Transportation Systems (ITSC), Rio de Janeiro, Brazil, 1-4 November 2016; pp. 1798-1804.

25. Tian, Z.; Cai, Y.; Huang, S.; Hu, F.; Li, Y.; Cen, M. Vehicle tracking system for intelligent and connected vehicle based on radar and V2V fusion. In Proceedings of the 2018 Chinese Control And Decision Conference (CCDC), Shenyang, China, 9-11 June 2018; pp. 6598-6603.

26. Noh, S.; An, K. Decision-Making Framework for Automated Driving in Highway Environments. IEEE Trans. Intell. Transp. Syst. 2017, PP, 1-14. [CrossRef]

27. Meng, F.; Su, J.; Liu, C.; Chen, W.H. Dynamic decision making in lane change: Game theory with receding horizon. In Proceedings of the 2016 UKACC 11th International Conference on Control (CONTROL), Belfast, UK, 31 August-2 September 2016.

28. Kala, R.; Warwick, K. Motion Planning of Autonomous Vehicles in A Non-autonomous Vehicle Environment without Speed Lanes. Int. J. Eng. Appl. Artif. Intell. 2013, 26, 1588-1601. [CrossRef]

29. Gonzalez, D.; Perez, J. Control Architecture for Cybernetic Transportation Systems in Urban Environments. In Proceedings of the 2013 IEEE Intelligent Vehicles Symposium (IV), Gold Coast, Australia, 23-26 June 2013; pp. 1119-1124.

30. Kurt, A. Discrete-State Encoding in Hybrid-State Systems for Intelligent Vehicle Control and Estimation. IEEE Trans. Intell. Transp. Syst. 2015, 16, 1595-1600. [CrossRef]

31. Zambrano-Martinez, J.L.; Calafate, C.T.; Soler, D.; Cano, J.C.; Manzoni, P. Modeling and Characterization of Traffic Flows in Urban Environments. Sensors 2018, 18, 2020. [CrossRef] [PubMed]

32. Furda, A.; Vlacic, L. Enabling Safe Autonomous Driving in Real-World City Traffic using Multiple Criteria Decision Making. IEEE Trans. Intell. Transp. Syst. 2011, 3, 4-17. [CrossRef]

33. Hubmann, C.; Becker, M.; Althoff, D.; Lenz, D.; Stiller, C. Decision making for autonomous driving considering interaction and uncertain prediction of surrounding vehicles. In Proceedings of the 2017 IEEE Intelligent Vehicles Symposium (IV), Los Angeles, CA, USA, 11-14 June 2017; pp. 1671-1678.

34. Kim, K. Collision Free Autonomous Ground Traffic: A Model Predictive Control Approach. In Proceedings of the 2013 ACM/IEEE International Conference on Cyber-Physical Systems (ICCPS), Philadelphia, PA, USA, 8-11 April 2013; pp. 51-60.

35. Weiskircher, T.; Wang, Q.; Ayalew, B. Predictive Guidance and Control Framework for (Semi-)Autonomous Vehicles in Public Traffic. IEEE Trans. Control Syst. Technol. 2015, 25, 2034-2046. [CrossRef]

36. Rafaila, R.; Caruntu, C.; Livint, G. Centralized Model Predictive Control of Autonomous Driving Vehicles with Lyapunov Stability. In Proceedings of the 2016 IEEE 20th International Conference on System Theory, Control and Computing (ICSTCC), Sinaia, Romania, 13-15 October 2016; pp. 663-668.

37. Koga, A.; Okuda, H.; Tazaki, Y.; Suzuki, T.; Haraguchi, K.; Kang, Z. Realization of Different Driving Characteristics for Autonomous Vehicle by using Model Predictive Control. In Proceedings of the 2016 IEEE Intelligent Vehicles Symposium (IV), Gothenburg, Sweden, 19-22 June 2016; pp. 722-728. 
38. Rosolia, U.; Bruyne, S.; Alleyne, A. Autonomous Vehicle Control: A Nonconvex Approach for Obstacle Avoidance. IEEE Trans. Control Syst. Technol. 2017, 25, 469-484. [CrossRef]

39. Beaucorps, P.D.; Streubel, T.; Verroust-Blondet, A.; Nashashibi, F.; Bradai, B.; Resende, P. Decision-making for automated vehicles at intersections adapting human-like behavior. In Proceedings of the 2017 IEEE Intelligent Vehicles Symposium (IV), Los Angeles, CA, USA, 11-14 June 2017.

40. Aoki, S.; Rajkumar, R.R. A configurable synchronous intersection protocol for self-driving vehicles. In Proceedings of the 2017 IEEE 23rd International Conference on Embedded and Real-Time Computing Systems and Applications (RTCSA), Hsinchu, Taiwan, 16-18 August 2017; pp. 1-11.

41. Santa, J.; Pereniguez, F.; Moragon, A.; Skarmeta, A.F. Vehicle-to-infrastructure Messaging Proposal Based on CAM/DENM Specifications. In Proceedings of the 2013 IEEE IFIP Wireless Days (WD), Valencia, Spain, 13-15 November 2013; pp. 1-7.

42. Zohdy, I.H.; Kamalanathsharma, R.K.; Rakha, H. Intersection Management for Autonomous Vehicles using iCACC. In Proceedings of the 2012 IEEE 15th Conference on Intelligent Transportation Systems (ITSC), Anchorage, AK, USA, 16-19 September 2012; pp. 1109-1114.

43. Tiganasu, A.; Lazar, C.; Caruntu, C.F. Design and simulation evaluation of cooperative adaptive cruise control for a platoon of vehicles. In Proceedings of the 2016 20th International Conference on System Theory, Control and Computing (ICSTCC), Sinaia, Romania, 13-15 October 2016; pp. 669-674.

44. Hafner, M.R.; Cunningham, D.; Caminiti, L.; Vecchio, D.D. Cooperative Collision Avoidance at Intersections: Algorithms and Experiments. IEEE Trans. Veh. Technol. 2013, 14, 1162-1175. [CrossRef]

45. Mladenovic, M.N.; Abbas, M.M. Self-organizing Control Framework for Driverless Vehicles. In Proceedings of the IEEE 16th International Conference on Intelligent Transportation Systems (ITSC), The Hague, The Netherlands, 6-9 October 2013; pp. 2076-2081.

46. Liu, B.; Kamel, A.E. V2X-Based Decentralized Cooperative Adaptive Cruise Control in the Vicinity of Intersections. IEEE Trans. Intell. Transp. Syst. 2016, 17, 644-658. [CrossRef]

47. Campos, G.R.D.; Falcone, P.; Hult, R.; Wymeersch, H.; Sjöberg, J. Traffic coordination at road intersections: Autonomous decision-making algorithms using model-based heuristics. IEEE Intell. Transp. Syst. Mag. 2017, 9, 8-21. [CrossRef]

48. Xu, B.; Li, S.E.; Bian, Y.; Li, S.; Ban, X.J.; Wang, J.; Li, K. Distributed conflict-free cooperation for multiple connected vehicles at unsignalized intersections. Transp. Res. Part C Emerg. Technol. 2018, 93, 322-334. [CrossRef]

49. Elleuch, I.; Makni, A.; Bouaziz, R. Cooperative Intersection Collision Avoidance Persistent System Based on V2V Communication and Real-Time Databases. In Proceedings of the 2017 IEEE/ACS 14th International Conference on Computer Systems and Applications (AICCSA), Hammamet, Tunisia, 30 October-3 November 2017; pp. 1082-1089.

50. Li, J.; Dridi, M.; El-Moudni, A. A cooperative traffic control for the vehicles in the intersection based on the Genetic Algorithm. In Proceedings of the 2016 IEEE 4th International Colloquium on Information Science and Technology (CiSt), Tangier, Morocco, 24-26 October 2017; pp. 627-632.

51. Ran, B.; Boyce, D. Dynamic Urban Transportation Network Models: Theory and Implications for Intelligent Vehicle-Highway Systems, 1st ed.; Springer: Berlin, Germany, 2012.

52. Ren, X.P.; Cai, Z.X. Using the Ackerman Principle for Kinematic Modeling of Wheeled Mobile Robots. CAA Trans. Intell. Syst. 2009, 4, 534-537.

53. Simionescu, P.A.; Beale, D. Optimum Synthesis of the Fourbar Function Generator in its Symmetric Embodiment: the Ackermann Steering Linkage. Int. J. Mech. Mach. Theory 2002, 37, 1487-1504. [CrossRef]

54. Kanagaraj, V.; Asaithambi, G.; Kumar, C.; Srinivasan, K.; Sivanandan, R. Evaluation of Different Vehicle Following Models under Mixed Traffic Conditions. Int. J. Procedia-Soc. Behav. Sci. 2013, 104, 390-401. [CrossRef]

55. Behrisch, M.; Bieker, L.; Erdmann, J.; Krajzewicz, D. SUMO—Simulation of Urban Mobility: An Overview. In Proceedings of the Third International Conference on Advances in System Simulation, ThinkMind (SIMUL2011), Barcelona, Spain, 23-29 October 2011; pp. 55-60.

56. Varga, A.; Hornig, R. An Overview of the OMNeT++ Simulation Environment. In Proceedings of the 1st International Conference on Simulation Tools and Techniques for Communications, Networks and Systems \& Workshops, Marseille, France, 3-7 March 2008; p. 60. 
57. Debada, E.; Makarem, L.; Gillet, D. A virtual vehicle based coordination framework for Autonomous Vehicles in heterogeneous scenarios. In Proceedings of the 2017 IEEE International Conference on Vehicular Electronics and Safety (ICVES), Vienna, Austria, 27-28 June 2017.

58. Dos Santos, T.C.; Gómez, A.E.; Filho, C.M.; Gomes, D.; Perafan, J.C.; Wolf, D.F.; Osório, F.; Rosero, L.A. A Simulation Framework for Multi-Vehicle Communication. In Proceedings of the 2015 12th Latin American Robotics Symposium and 2015 3rd Brazilian Symposium on Robotics (LARS-SBR), Uberlandia, Brazil, 29-31 October 2015; pp. 301-308.

59. Tang, W.; Yang, M.; Lyu, Z.; Qian, Q.; Su, T.; Wang, B.; Wang, C. MicroIV: A Cooperative Driving Hardware Simulation Platform for Cooperative-ITS. IEEE Trans. Veh. Technol. 2018, 1. [CrossRef]

60. Chen, X.; Miao, Y. Driving Decision-Making Analysis of Car-Following for Autonomous Vehicle under Complex Urban Environment. In Proceedings of the 2016 9th International Symposium on Computational Intelligence and Design (ISCID), Hangzhou, China, 10-11 December 2016; Volume 1, pp. 315-319.

61. Artuñedo, A.; Godoy, J.; Haber, R.; Villagrá, J.; Toro, R.M.D. Advanced Co-simulation Framework for Cooperative Maneuvers Among Vehicles. In Proceedings of the 2015 IEEE 18th International Conference on Intelligent Transportation Systems (ITSC), Gran Canaria, Spain, 15-18 September 2015; pp. 1436-1441.

62. Kim, H.; Park, J.; Kim, D.; Youn, C.H.; Han, J.; Kim, S.H.; Choi, J.; Yoon, D.; Jeon, M.; Yang, E. IsV2C: An Integrated Road Traffic-Network-Cloud Simulator for V2C Connected Car Services. In Proceedings of the 2017 IEEE International Conference on Services Computing (SCC), Honolulu, HI, USA, 25-30 June 2017; pp. 434-441.

63. Fok, C.; Hanna, M.; Gee, S.; Au, T.; Stone, P.; Julien, C.; Vishwanath, S. A Platform for Evaluating Autonomous Intersection Management Policies. In Proceedings of the 2012 IEEE/ACM 3rd International Conference on Cyber-Physical Systems (ICCPS), Beijing, China, 17-19 April 2012; pp. 87-96.

64. Gupta, N.; Vijay, R.; Korupolu, P.; Bansal, J.; Kapuria, A. Architecture of Autonomous Vehicle Simulation and Control Framework. In Proceedings of the 2015 ACM Conference on Advances In Robotics (AIR), Goa, India, 2-4 July 2015; pp. 1-6.

(c) 2018 by the authors. Licensee MDPI, Basel, Switzerland. This article is an open access article distributed under the terms and conditions of the Creative Commons Attribution (CC BY) license (http:/ / creativecommons.org/licenses/by/4.0/). 